\title{
Gouvernance territoriale de l'environnement et conflits d'usage. Le cas du bassin versant du lac de Tota (Boyacá, Andes colombiennes)
}

David Leroy, Alvaro Martín Gutiérrez Malaxechebaría, Jean-Marc Antoine and Alexandra Angéliaume-Descamps

\section{(2) OpenEdition}

Journals

Electronic version

URL: https://journals.openedition.org/echogeo/15238

DOI: 10.4000/echogeo.15238

ISSN: 1963-1197

Publisher

Pôle de recherche pour l'organisation et la diffusion de l'information géographique (CNRS UMR 8586)

\section{Electronic reference}

David Leroy, Alvaro Martín Gutiérrez Malaxechebaría, Jean-Marc Antoine and Alexandra AngéliaumeDescamps, "Gouvernance territoriale de l'environnement et conflits d'usage. Le cas du bassin versant du lac de Tota (Boyacá, Andes colombiennes)", EchoGéo [Online], 43 | 2018, Online since 22 March 2018, connection on 23 August 2021. URL: http://journals.openedition.org/echogeo/15238; DOI: https://doi.org/10.4000/echogeo.15238

\section{This text was automatically generated on 23 August 2021}

EchoGéo est mis à disposition selon les termes de la licence Creative Commons Attribution - Pas d'Utilisation Commerciale - Pas de Modification 4.0 International (CC BY-NC-ND) 


\title{
Gouvernance territoriale de l'environnement et conflits d'usage. Le cas du bassin versant du lac de Tota (Boyacá, Andes colombiennes)
}

\author{
David Leroy, Alvaro Martín Gutiérrez Malaxechebaría, Jean-Marc Antoine \\ and Alexandra Angéliaume-Descamps
}

\section{Introduction}

1 Depuis quelques décennies, la gestion de l'environnement est devenue un élément essentiel des dispositifs de gouvernance des territoires (Theys, 2002 ; Beaurain, 2002 ; Hinnewinkel et Guillerme, 2004; Froger, 2008; Scarwell et al., 2008; Young, 2009; Beuret et Cadoret, 2011). Au Nord comme au Sud, se sont mis en place différents processus de décision et de concertation entre des acteurs de statuts différents (producteurs, associations, particuliers, pouvoirs publics...) afin de chercher à encadrer les externalités environnementales des activités productives (surexploitation des ressources, pollution), de prévenir les risques environnementaux et sanitaires (accès à l'eau, déclin de la biodiversité...), ou encore de préserver et protéger les espaces naturels fragiles (Salles et Leroy, 2013).

2 Si la prise en compte des questions environnementales a permis à la société de reconsidérer ses territoires, notamment via l'émergence de nouvelles modalités d'organisation entre les acteurs, cette forme de gouvernance ne va pas de soi. Elle est faite « de phases de négociation, de collaboration ou d'apaisement, mais également de périodes beaucoup plus animées, ou conflictuelles, au cours desquelles certains groupes ou catégories d'acteurs s'opposent, parfois avec violence, pour définir les marches à suivre et les options à retenir » (Torre, 2015).

3 La gouvernance territoriale de l'environnement, qui s'entend comme l'ensemble des modes de coordination et d'organisation des acteurs destinés à gérer les ressources 
naturelles et l'environnement d'un territoire, ne se limite donc pas aux processus de décision permettant la régulation d'un problème commun. "Il s'agit également d'une interaction entre des forces poussant à la coopération et d'autres forces, qui poussent au conflit» (Torre, 2011a). Ainsi, à l'image des travaux d'André Torre (2011a, 2011b, 2015), nous faisons l'hypothèse que les conflits jouent un rôle essentiel dans les mécanismes de gouvernance territoriale de l'environnement, en contribuant à la prise en compte des problèmes environnementaux et à l'expression démocratique des oppositions.

4 De ce fait, les interactions entre la gouvernance territoriale, les problèmes environnementaux et les conflits d'usages constituent aujourd'hui une question centrale du développement des territoires ruraux, en raison de leur caractère multifonctionnel. En effet, longtemps restreints à la seule fonction économique sectorielle et productiviste, les territoires ruraux servent aujourd'hui de support à de nouvelles activités telles la récréation, la résidence ou encore la conservation de l'environnement. Toutefois, cette multifonctionnalité induit par nature des usages concurrents, et, de ce fait, des divergences et des oppositions entre les différents acteurs (Torre et al., 2006).

5 Ainsi, dans les Andes colombiennes, et en particulier au sein du bassin versant du lac de Tota, on assiste depuis plusieurs années à des conflits entre les communautés rurales, les institutions environnementales (CORPOBOYACÁ) et une association environnementale (Montecito) autour de la gestion des conséquences environnementales de l'agriculture intensive. Principale activité économique de la région, et notamment du municipe d'Aquitania où se concentre l'essentiel de la production, la culture de la ciboule a permis de dynamiser économiquement ce territoire. Toutefois, elle s'accompagne d'une dégradation environnementale d'envergure, liée à la surexploitation et à la contamination biochimique de l'eau du lac, à laquelle s'ajoutent les pratiques intensives des pisciculteurs, des populations villageoises et du secteur hôtelier (rejet des eaux usées dans le lac), ou encore de l'industrie (pompage de l'eau du lac).

6 Ce travail porte donc sur les conflits liés à la gestion des problèmes environnementaux dans le bassin versant du lac de Tota (Andes colombiennes). Il vise plus particulièrement à démontrer que ces conflits font partie intégrante des processus de gouvernance territoriale de l'environnement. Nous nous appuierons tout particulièrement sur 60 entretiens semi-directifs avec les principaux acteurs impliqués dans la gouvernance du lac (agriculteurs, institutions environnementales, secteur touristique, associations environnementales...) sur l'analyse des outils et mécanismes de gouvernance (plans d'aménagement, instruments financiers, législations, instances de coordination ...), ainsi que sur l'observation participante mise en œuvre lors d'une mission sur le terrain réalisée en 2014-2015.

7 Nous présenterons dans un premier temps le terrain d'étude, le bassin versant du lac de Tota, support de multiples fonctions économiques, sociales et environnementales. Il s'agira plus particulièrement d'étudier l'impact des activités économiques, et notamment de l'agriculture intensive, sur l'environnement du lac. Dans un second temps, à travers l'analyse de plusieurs situations concrètes, nous démontrerons que les conflits liés à la gestion des problèmes environnementaux contribuent à l'expression des désaccords, et, de fait, aux processus de gouvernance territoriale de 
l'environnement. Enfin, nous montrerons que malgré une période d'avancée et d'innovation, la gouvernance reste un enjeu de pouvoir.

\section{Le lac de Tota : un espace multifonctionnel menacé}

Espace de production, hydro-écosystème stratégique, ressource en eau, paysage, patrimoine culturel... le bassin versant ${ }^{1}$ du lac de Tota est un espace multifonctionnel, mobilisant une pluralité d'acteurs. Si certaines activités sont orientées vers la conservation de la qualité et l'intégrité environnementales du bassin versant, d'autres, au contraire, vont être à l'origine d'une dégradation considérable de l'environnement.

\section{Entre biodiversité, paysage, patrimoine culturel et ressource en eau}

9 Le lac de Tota (illustration 1) est le plus grand lac de Colombie; il a une superficie de 6000 ha et un volume de stockage de 1900 millions de mètres cubes (DNP, 2014). Situé dans la cordillère orientale des Andes colombiennes, à $3015 \mathrm{~m}$ d'altitude, il est mondialement reconnu pour sa biodiversité et les paysages exceptionnels qu'il génère, mais aussi pour son rôle stratégique dans l'alimentation en eau des populations andines colombiennes. Ce lac approvisionne plus de 250000 personnes, soit environ $20 \% \mathrm{du}$ total de la population du département de Boyacá. Toutefois, la majorité des villes ${ }^{2}$ et villages qu'il alimente se situe en aval, hors de son bassin versant (DNP, 2014). D'origine glaciaire, le lac de Tota est bordé par les municipalités d'Aquitania, Cuítiva, Tota et Sogamoso. Son bassin versant s'étend sur 22380 ha (illustration 2).

Illustration 1 - Le lac de Tota

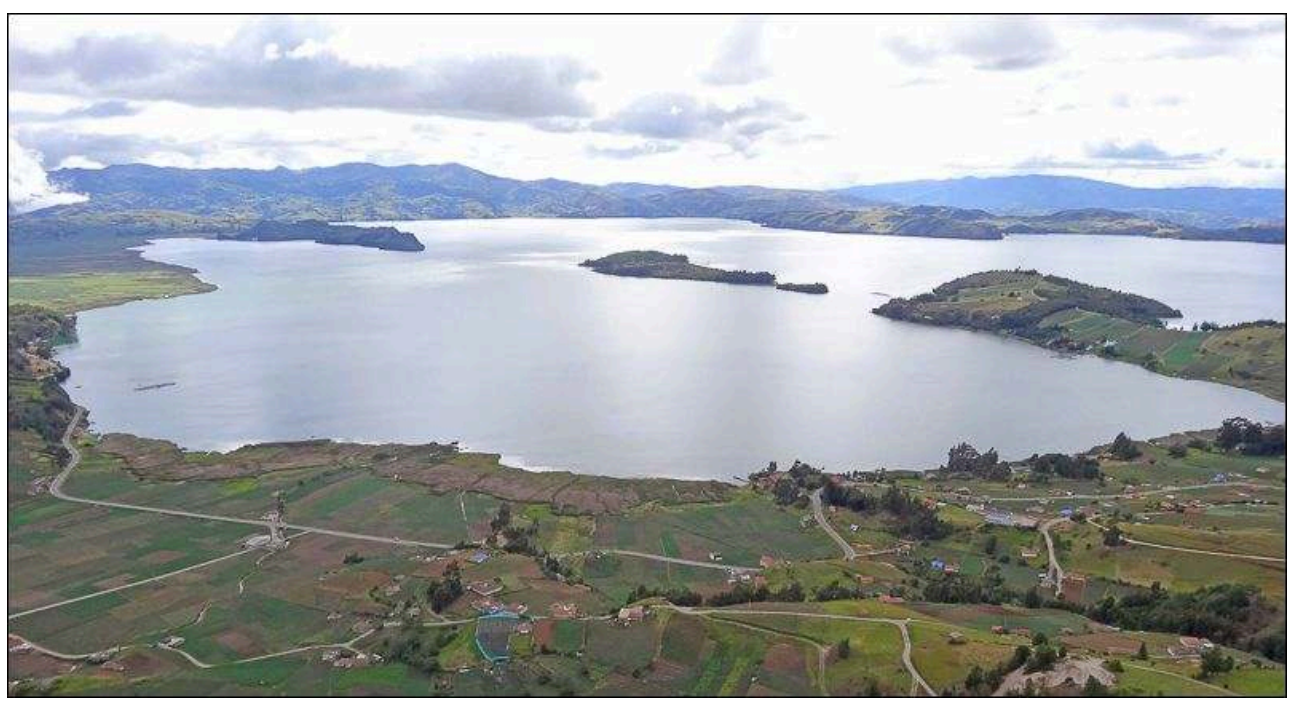

Auteur : D. Leroy, 2014. 
Illustration 2 - Situation géographique du bassin versant du lac de Tota

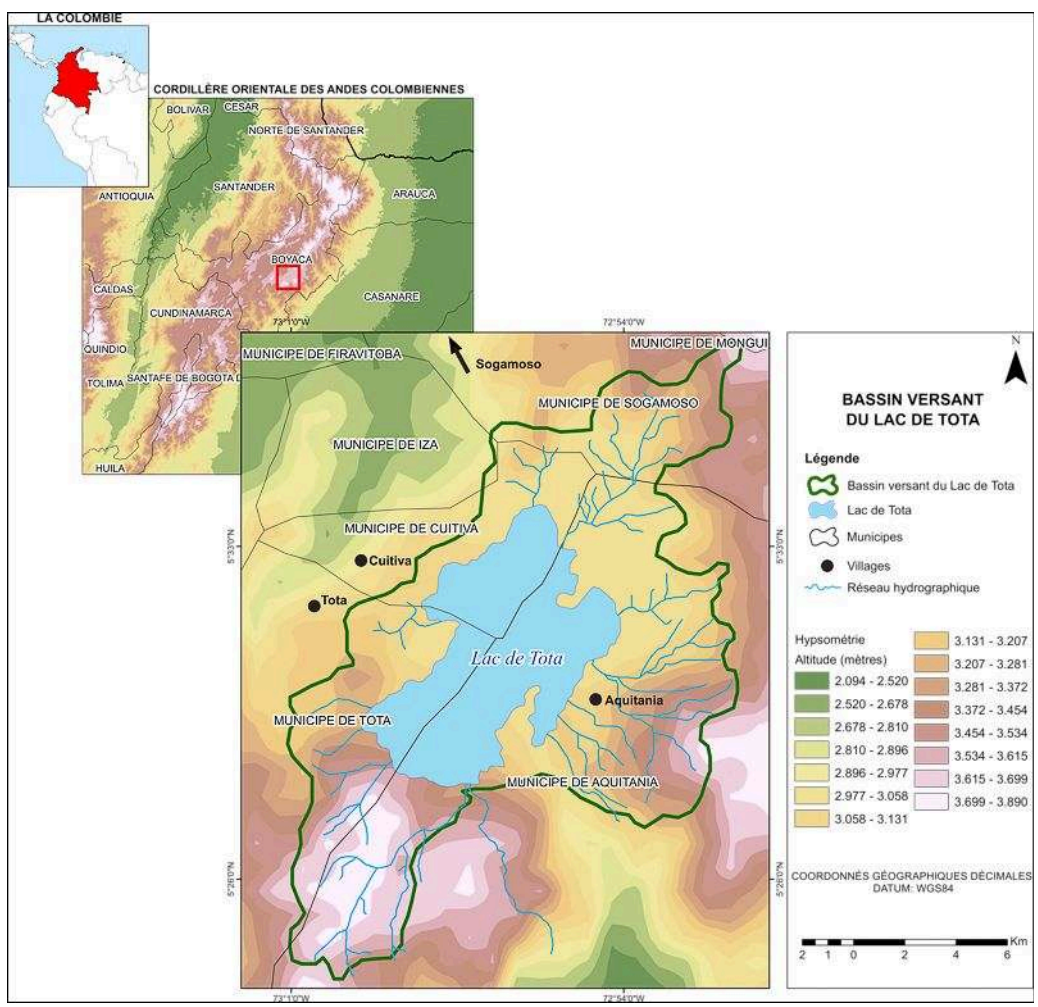

Auteurs : E. P. Tapiquén et D. Leroy, 2016.

Du fait des enjeux sociaux et environnementaux, le bassin versant du lac de Tota fait l'objet d'une protection spéciale liée à la loi 99 de $1993^{3} \mathrm{du}$ Ministère de l'environnement colombien. Car en plus d'être la principale ressource hydrique de la région, il se situe dans le páramo, écosystème d'altitude associé à l'étage andin, spécifique des Andes septentrionales (Colombie, Équateur, Venezuela et nord du Pérou). Comme tout páramo, le páramo de Tota-Bijagual-Mamapacha est un écosystème essentiel pour la régulation du cycle de l'eau. Son altitude en fait en effet un espace fondamental de captation des eaux de pluie et de formation d'une multitude de sources, qui alimentent une grande partie (57\%) du bassin versant du lac (DNP, 2014), soit directement par ruissellement superficiel vers les quebradas, petits cours d'eau de montagnes, soit en transitant par des lagunas, petits lacs d'origine glaciaire, ou diverses autres zones humides.

Ce complexe humide est par ailleurs considéré comme faisant partie des aires de conservation des oiseaux des Andes tropicales. Il héberge une riche avifaune endémique et sédentaire et fait partie des viviers importants pour certaines espèces d'oiseaux migrateurs (Pluviales dominica, Actitis macularia...) (Niño et al., 2011 ; DNP, 2014). On y rencontre également des espèces végétales endémiques, tel que le frailejón, espèce emblématique du páramo. Il n'est donc pas étonnant que cet espace intéresse de très près le monde académique, en particulier les universités de Boyacá et de Bogota. En effet, depuis quelques décennies, le lac de Tota est l'objet de nombreuses études universitaires d'horizons disciplinaires très variées (écologie, climatologie, géographie, sociologie...). Parallèlement aux activités liées à la recherche scientifique, l'Université joue également un rôle important dans l'éducation environnementale. Elle vient ainsi s'associer aux nombreuses ONG et associations environnementales (Fundación Montecito, 
ONG Tejido Ambiente, ONG Fundación Amigos Pro Desarrollo municipio de Cuítiva y Defensa del Lago de Tota, Futuro Verde ONG Ambientalista Lago de Tota) qui militent pour la conservation du lac.

12 Le principal acteur de la gestion du bassin versant du lac de Tota est sans conteste la Corporación Autónoma Regional de Boyacá (CORPOBOYACÁ). En effet, depuis 1993, la CORPOBOYACÁ est chargée «d'administrer l'environnement et les ressources naturelles renouvelables [du bassin versant $d u l a c]$, ainsi que d'œuvrer à son développement durable, en conformité avec les dispositions légales et les politiques du Ministère de l'Environnement ». La CORPOBOYACÁ est notamment venue succéder à la CAR $^{4}$ (Corporación Autónoma Regional), créée en 1973, en tant qu'institution environnementale. Bien que rattachée au Ministère de l'Environnement, cette corporation est dotée d'une autonomie administrative et financière, et d'un statut juridique mandaté par la loi. La CORPOBOYACÁ s'appuie notamment sur un plan d'aménagement afin de contrôler l'usage du sol et des ressources en eau. Il s'agit donc d'une autorité unique mise en place pour gérer et aménager le territoire.

Hydro-écosystème stratégique de la région de Boyacá, le bassin versant du lac de Tota constitue donc un patrimoine naturel que l'on préserve pour sa faune et sa flore emblématique et/ou endémique, mais aussi, et surtout, pour ses ressources en eau. Mais au-delà d'être un simple support biophysique, ce territoire possède également une valeur identitaire et culturelle unique. Le bassin versant du lac de Tota recèle en effet de nombreuses fêtes traditionnelles (San Pascual Bailón, Divino Niño...), mais il est aussi le berceau de plusieurs contes, mythes et légendes (le fameux monstre du lac de Tota). Le savoir-faire lié à l'artisanat traditionnel d'Aquitania est également reconnu dans toute la Colombie. Chaque habitant de cet espace alto andin possède en effet sa juana, vêtement traditionnel des Andes du Nord, qui constitue la norme vestimentaire. Mais la caractéristique identitaire la plus remarquable du bassin versant est sans conteste celle de la culture de ciboule, car Aquitania est aujourd'hui la capitale nationale de la ciboule.

\section{La « révolution » de la ciboule : vers l'intensification de l'agriculture irriguée}

14 Le bassin versant du lac de Tota est habité principalement par une population métisse, descendante du brassage entre Espagnols et Indiens, dont l'activité principale est l'agriculture. Les paysans sont essentiellement de petits propriétaires terriens, exploitant de nombreuses parcelles de taille réduite (entre 0,5 et 5 ha) et dispersées (illustration 3). Aquitania est le seul village riverain du lac (illustration 4). Il accueille environ 6500 habitants qui dépendent directement du lac, aussi bien pour la consommation en eau, que pour l'agriculture. 
Illustration 3 - Petit paysan d'Aquitania travaillant son champ de ciboule

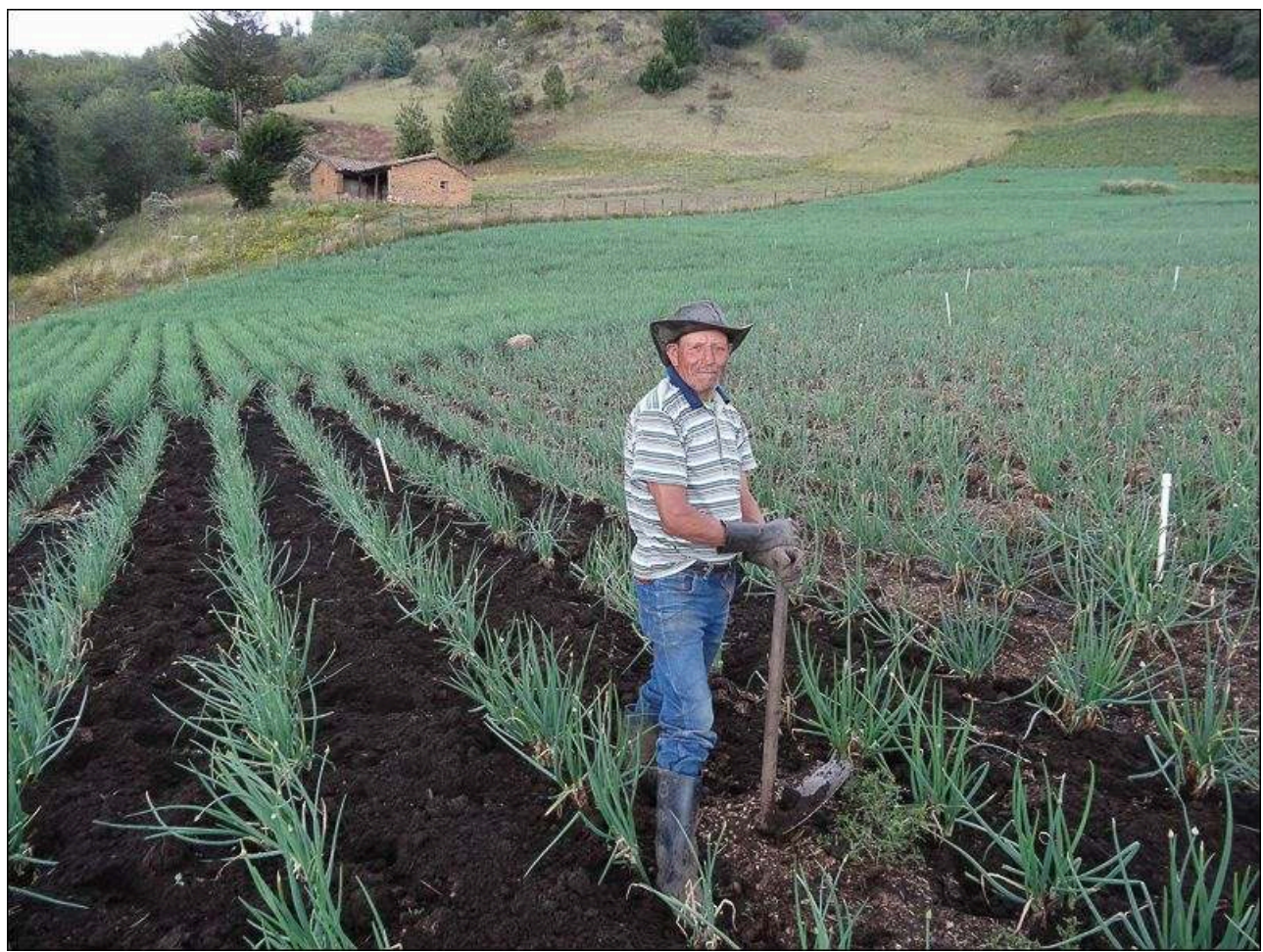

Auteur : D. Leroy, 2014

\section{Illustration 4 - Aquitania}

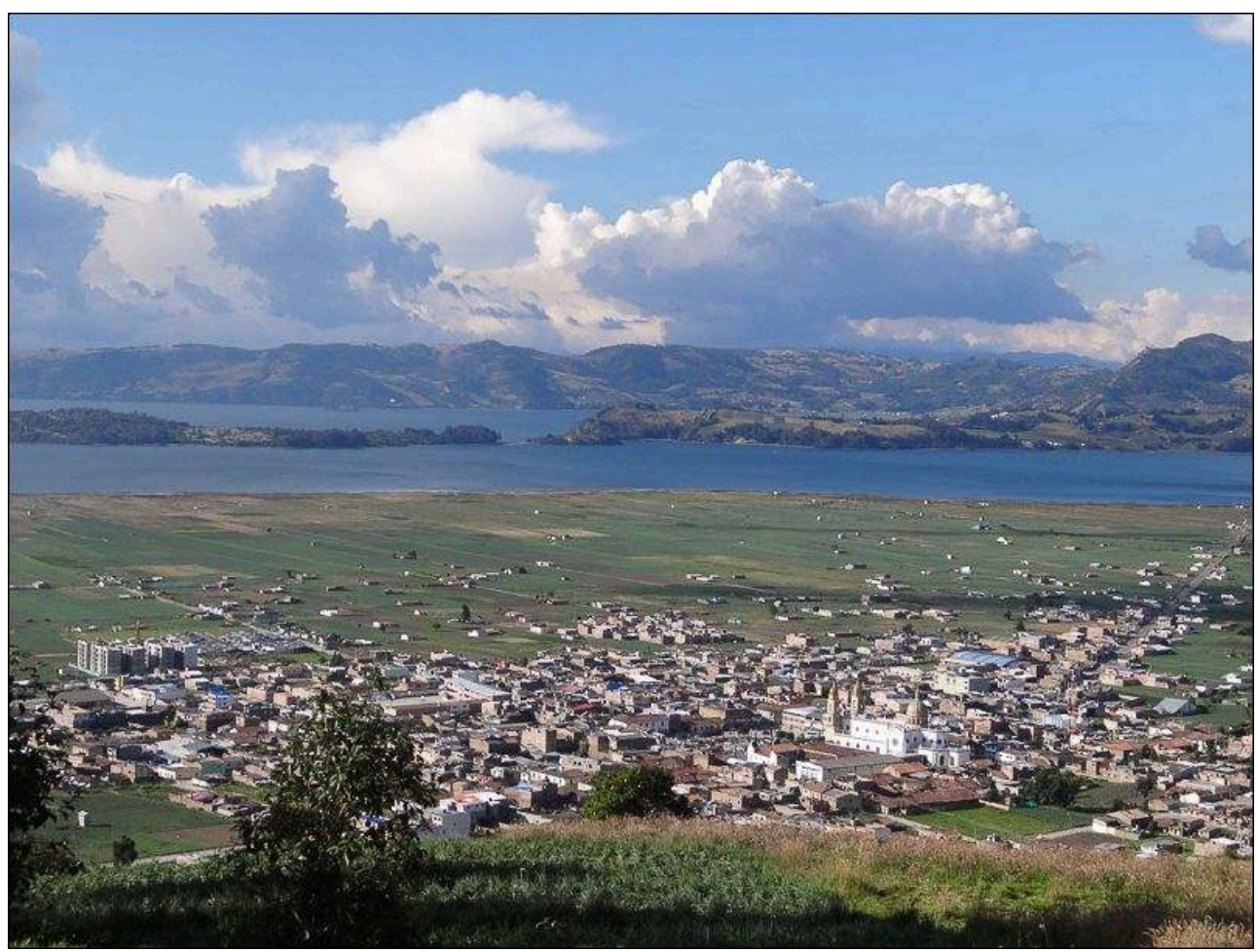

Au premier plan, le village d'Aquitania, au second plan, les cultures de ciboules qui s'étendent jusqu'au lac de Tota.

Auteur : D. Leroy, 2014. 
Dans les années 1950, les terres d'Aquitania étaient considérées comme des terroirs difficiles, contraignants. L'agriculture ne subvenait que partiellement aux besoins alimentaires de la population locale et ne s'exportait pas. La production agricole était alors caractérisée par une combinaison de différentes cultures traditionnelles (pommes de terre, orge, blé, fèves...), cultivées au rythme des saisons et en fonction des potentialités naturelles du milieu, notamment des sols, et de la conjoncture climatique.

Tout allait changer avec l'introduction d'une nouvelle culture commerciale : la ciboule. À cette époque, de nouvelles tendances alimentaires, notamment urbaines, apparaissent dans toute la Colombie, et la demande de ciboule devient de plus en plus forte. Il est vrai que ce condiment est particulièrement apprécié par les Colombiens, qui l'utilisent dans la plupart des plats, et plus particulièrement dans les soupes, notamment en raison de sa saveur piquante.

siboule devient rapidement une culture majeure dans la région, c'est avant tout parce qu'elle présente une rentabilité qui la rend particulièrement attractive pour les communautés rurales. Car contrairement à d'autres cultures plus traditionnelles telles que la pomme de terre, l'orge ou le blé, la ciboule est une culture semipermanente qui peut être semée à n'importe quelle époque de l'année, et qui a l'avantage de produire plusieurs récoltes (trois par an) à partir du moment où l'accès à l'eau est assuré.

D'autres zones en Colombie avaient déjà commencé à produire cette culture d'altitude, mettant en lumière sa rentabilité (Nariño, Norte de Santander...). Mais le facteur déterminant qui allait marquer une différence fondamentale avec les régions concurrentes réside dans la particularité, voire l'excellence, des conditions climatiques, hydriques et pédologiques des abords du lac de Tota : les sols d'Aquitania sont en effet particulièrement fertiles, le micro-climat généré par le lac idéal pour la croissance de l'allium, et l'eau suffisamment abondante pour satisfaire les besoins de la culture (Raymond, 1990). C'est pourquoi l'irrigation va être le principal outil de transformation agricole, allant souvent de pair avec l'application massive d'intrants. En quelques années, l'irrigation se généralise à l'ensemble des terres productives d'Aquitania (gravitaire sur les hauts versants et par pompage sur les zones riveraines du lac), ce qui entraîne une augmentation considérable de la production. En 2014, la superficie cultivée de ciboule (dont la grande majorité est irriguée) était de 2500 ha pour l'ensemble du bassin versant (DNP, 2014).

19 Aujourd'hui, la monoculture de la ciboule représente la principale activité économique $\mathrm{du}$ bassin versant du lac de Tota, dont dépendent directement et indirectement 16000 personnes (le nombre total de producteurs de ciboule est estimé à 4000 ). Selon les statistiques agricoles, Aquitania accueille même plus de $57 \%$ de la production nationale (MADR, 2014). On a donc affaire à une véritable agriculture industrielle qui alimente les principaux marchés du pays.

\section{Un territoire rural qui se diversifie}

Parallèlement, se développe depuis les années 1980 la pratique de la pisciculture en cage flottante (illustration 5). Ce système piscicole est essentiel pour l'économie de nombreuses familles d'Aquitania, de Cuítiva et de Tota. Il fournit plus de 650 tonnes de poissons par an, en particulier de truite Arco Iris. De ce fait, la région du lac de Tota est 
la première productrice de truite Arco iris du pays avec plus de $9 \%$ de la production nationale (Hidrósfera, 2012).

Illustration 5 - Pisiculture en cage flottante

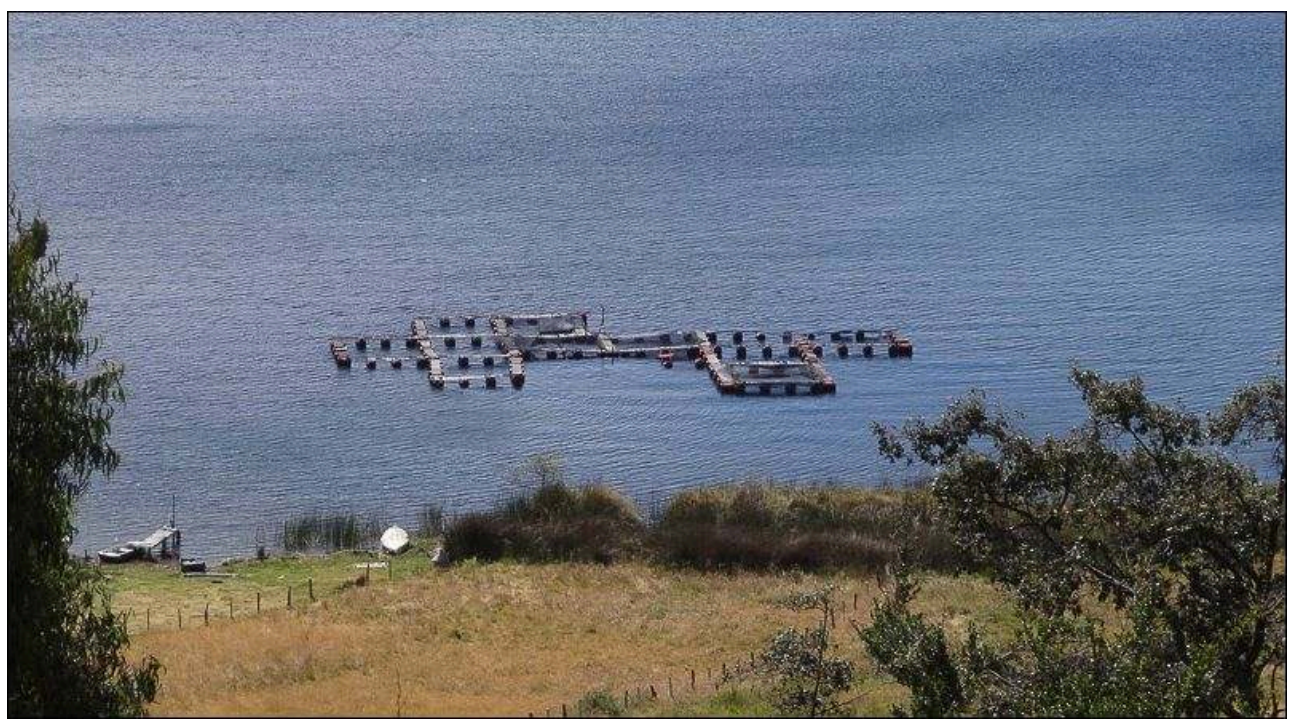

Auteur : D. Leroy, 2014

21 Bien que le secteur industriel ne soit pas particulièrement développé au sein du bassin versant, une multinationale sidérurgique, localisée à Sogamoso, Acerías Paz del Río, est l'un des plus gros consommateurs d'eau du lac de Tota (extraction de 200 litres d'eau par seconde) depuis 1952 , suite au décret $n^{\circ} 1111$ du gouvernement national lui permettant d'utiliser l'eau du lac à des fins industrielles (Pontificia Universidad Javeriana, 2005).

Enfin, bien que la dimension paysagère du lac de Tota soit de plus en plus valorisée, le secteur touristique n'est pas encore très développé. Seuls quelques restaurants et une dizaine d'hôtels bordent le lac. Mais en raison du fort potentiel qu'offrent le lac et ses environs, plusieurs projets sont en cours de développement, en particulier dans la zone de playa blanca (plage blanche, illustration 6), lieu très prisé par les touristes. 
Illustration 6 - Playa blanca, vitrine touristique du lac de Tota

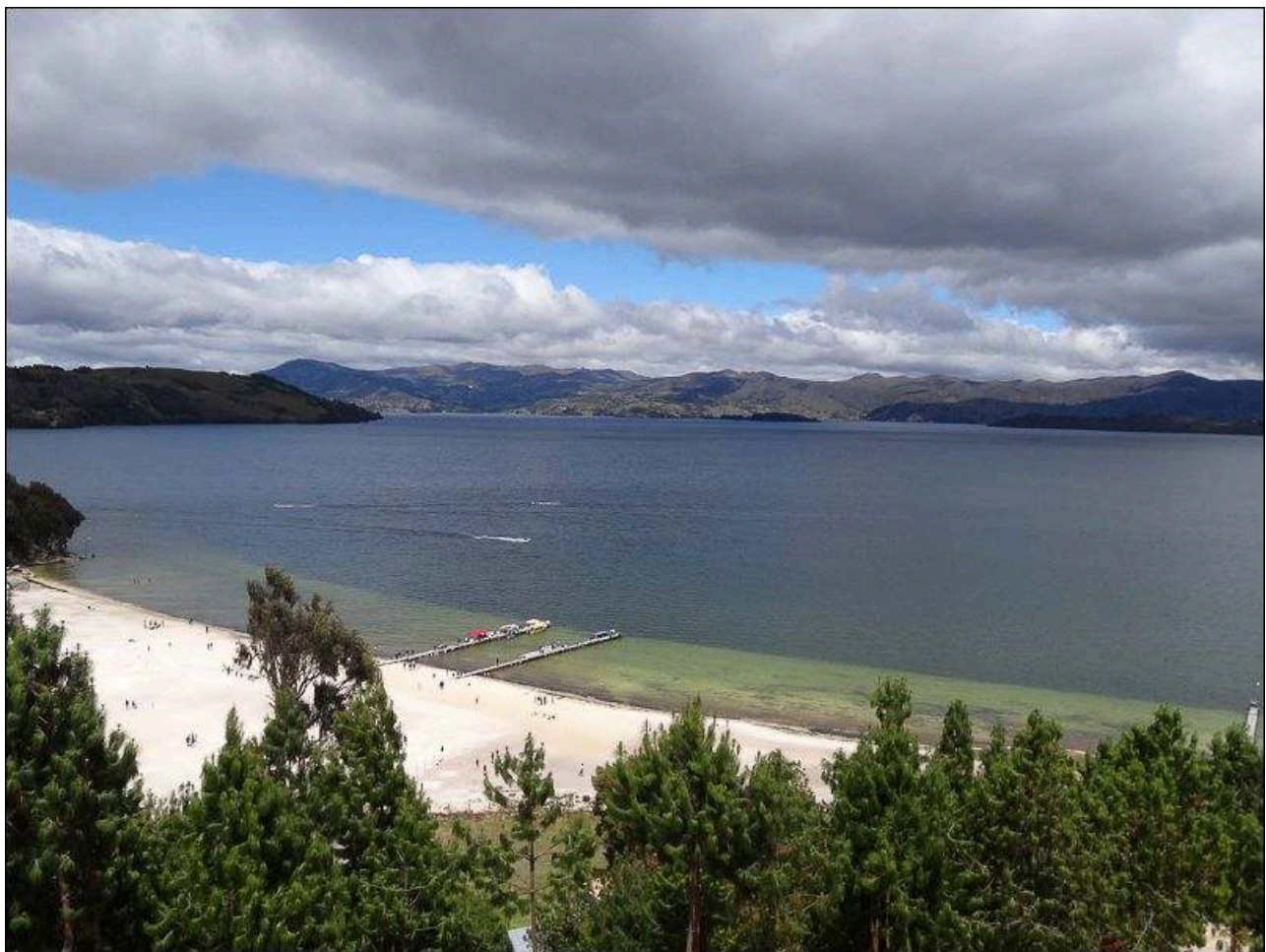

Auteur : D. Leroy, 2014

Il existe donc une grande diversité d'acteurs au sein du bassin versant du lac de Tota (illustration 7), et par conséquent une grande diversité d'intérêts liés à l'utilisation du lac et de ses ressources. Nous analyserons de manière plus approfondie les relations entre ces acteurs dans la seconde partie de cet article. 


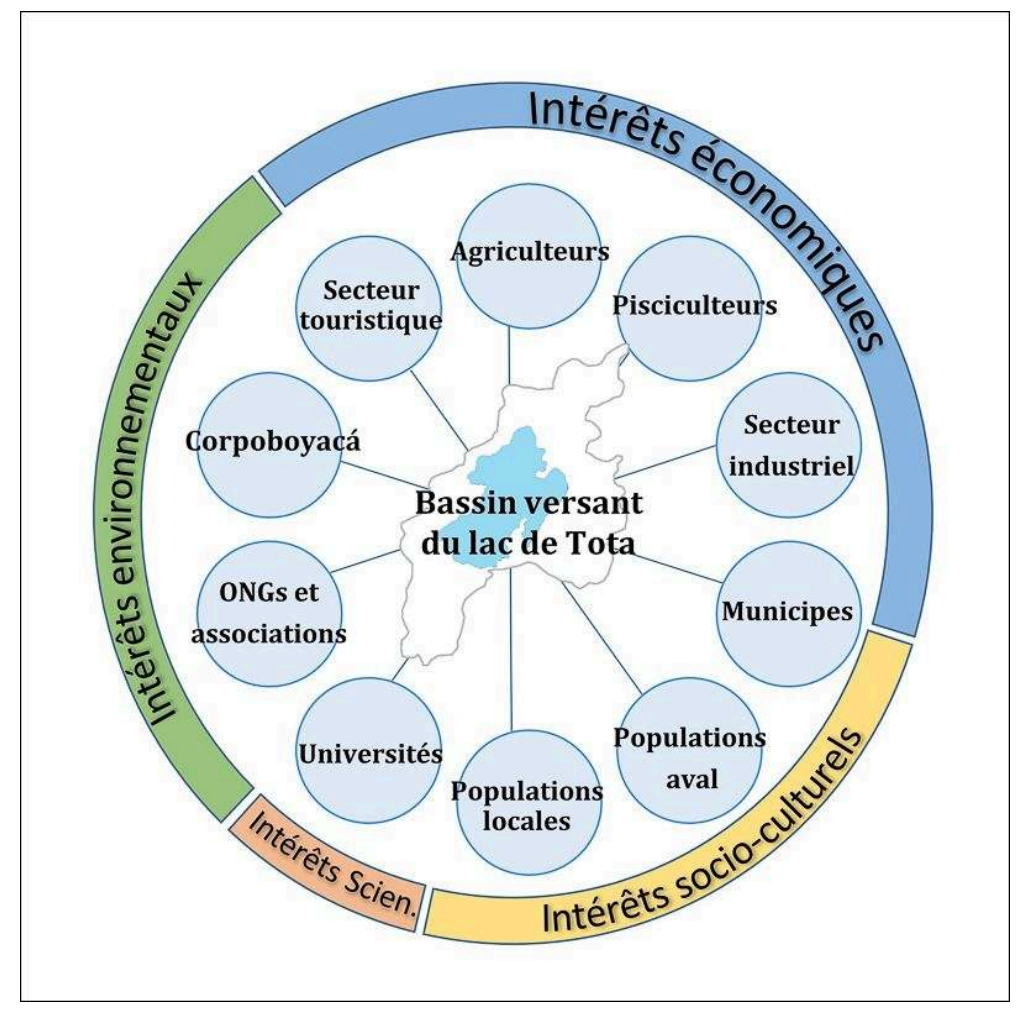

Auteur : D. Leroy, 2017.

\section{Une grande diversité de problèmes environnementaux}

Si le développement de plusieurs activités économiques, et en particulier la mise en place d'un système de production agricole hautement intensif, a permis de dynamiser l'économie de la région du lac de Tota, il s'accompagne en retour d'atteintes à l'environnement, notamment liées à une surexploitation et une contamination de l'eau du lac de Tota.

La surexploitation de l'eau est essentiellement liée à l'augmentation exponentielle des systèmes d'irrigation - par pompage - autour du lac. De plus, le développement de systèmes d'irrigation gravitaires sur les versants mobilise une part considérable de l'eau des tributaires de ce grand réservoir naturel. L'accès à la ressource est généralement synonyme de prospérité pour les agriculteurs. Elle permet d'intensifier la production de ciboule dans l'ensemble du bassin versant. L'expansion géographique de l'allium (illustration 8) ne se limite pas seulement aux versants surplombants, mais aussi aux rives mêmes du lac de Tota qui reculent de plusieurs centaines de mètres depuis les années 1960.

Par ailleurs, la remontée de la frontière agricole dans les páramos entraîne une réduction de la biodiversité, une banalisation des paysages et une altération considérable du cycle hydrologique. Ces espaces sont également soumis à une dynamique de mise en usage et d'augmentation des charges pastorales (bovines et ovines), ce qui endommage la végétation, compacte les sols et perturbe les fonctions de rétention en eau. 
Illustration 8 - Le bassin versant du lac de Tota, entre ressource en eau et espace de production

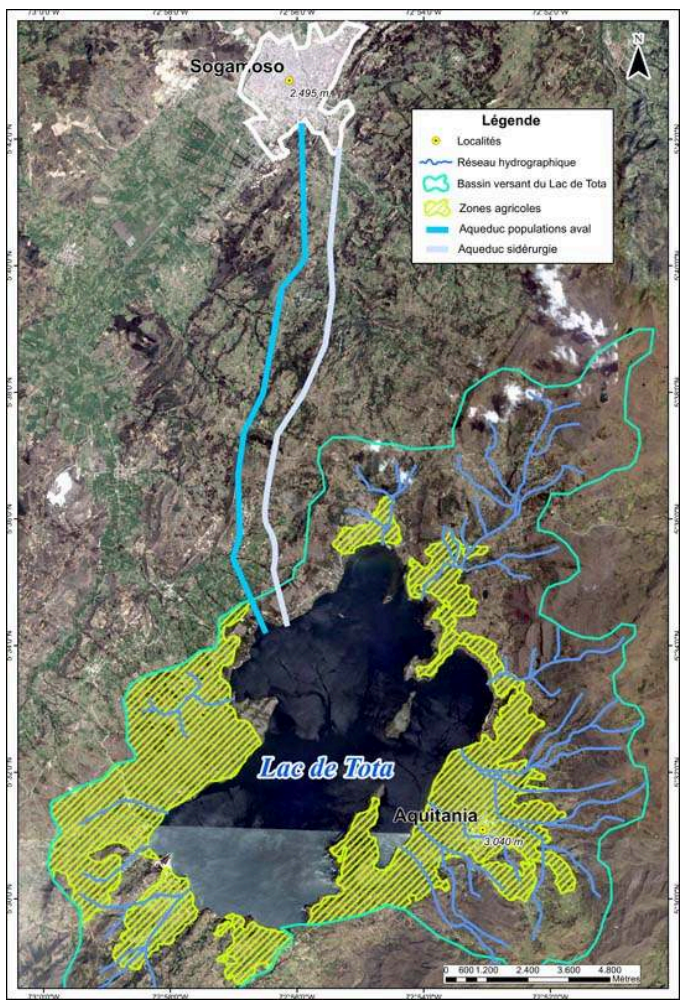

Auteur : E. P. Tapiquén et D. Leroy, 2017.

27 Soulignons également l'impact de l'industrie sidérurgique Acerías Paz del Río ainsi que des populations de l'aval (ville de Sogamoso, Iza, Nobsa...) qui mobilisent une quantité considérable de l'eau du lac, via deux aqueducs (illustration 8). Toutefois l'agriculture reste de loin le premier consommateur d'eau avec l'extraction de plus de 20 millions de mètres cubes d'eau sur un total de 41, 37 millions de mètres cubes (illustration 9) (Waves, 2016). 
Illustration 9 - Proportion de l'utilisation de l'eau au sein du bassin versant du lac de Tota en 2013 (eau du lac, des sources, cours d'eau et sol)

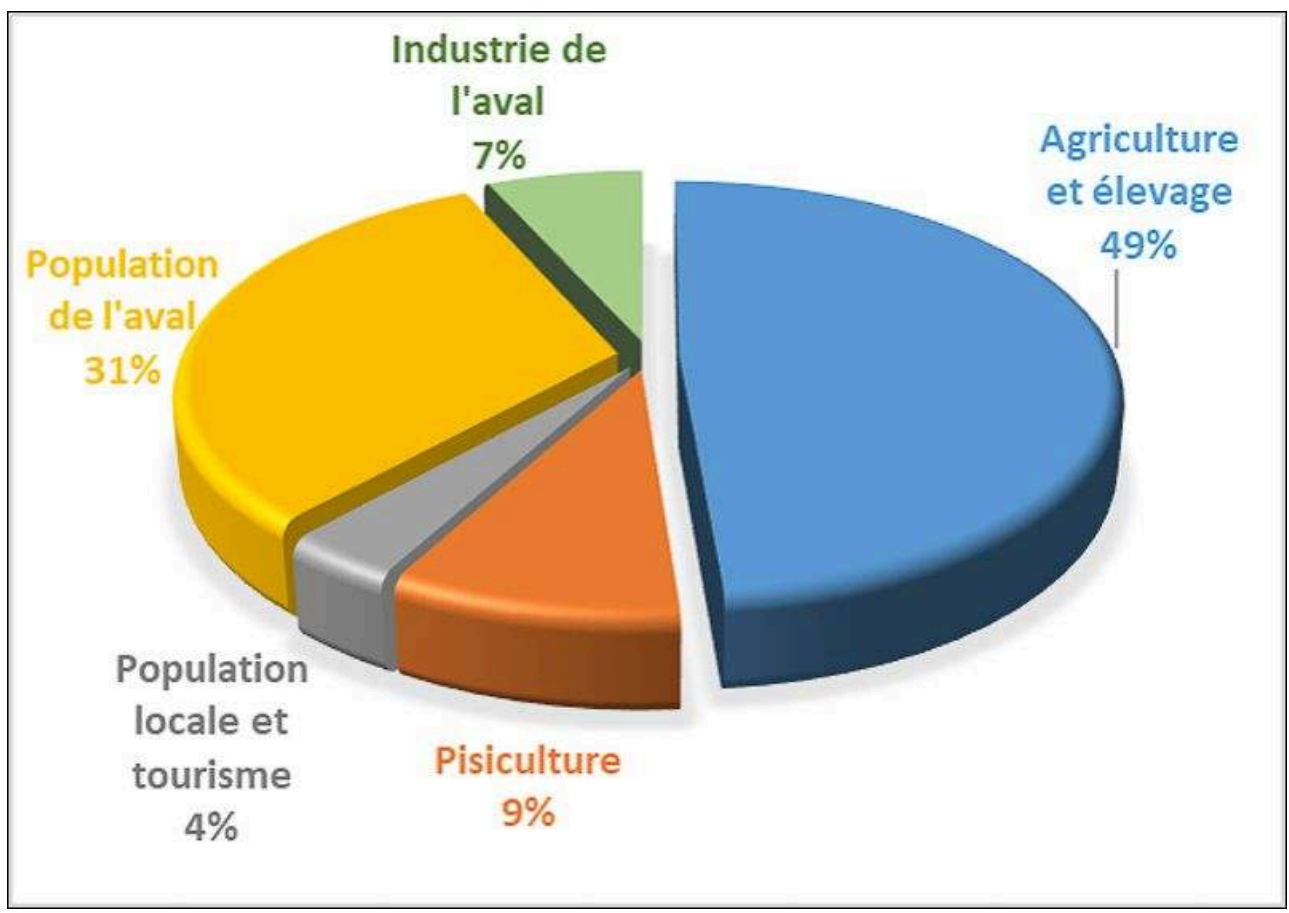

L'eau consommée par la pisciculture est en grande partie liée au lavage et à l'éviscération des truites. Source : Réalisation à partir des données de Waves, 2016.

Au-delà des problèmes liés à la surexploitation du lac, il est important de souligner que l'ensemble des populations (locales, en aval, agriculteurs, pisciculteurs...) est aussi potentiellement exposé aux risques de pollution des eaux de ce réservoir naturel, ce qui montre l'enjeu d'une gestion intégrée du territoire. La contamination est essentiellement due à l'utilisation massive de produits phytosanitaires, parfois très toxiques, ainsi qu'au recours généralisé à la fertilisation organique. C'est particulièrement le cas de la gallinaza, déjection de volaille, qui est utilisée par les agriculteurs pour enrichir les sols dégradés par plusieurs années de culture intensive. En plus d'être vectrice de problèmes pathogènes, la gallinaza contribue à l'eutrophisation du lac.

Bien que l'agriculture intensive soit la principale cause d'atteinte à l'environnement lacustre, d'autres activités ont également un impact considérable sur la santé de cet écosystème. C'est par exemple le cas de la pisciculture intensive qui rejette chaque année plus de 3, 4 tonnes de phosphore dans le lac (Valderrama, 2013), mais aussi de la population locale et du secteur hôtelier qui y déversent directement leurs eaux usées, sans aucun traitement, ajoutant au développement de l'eutrophisation.

Enfin, bien qu'étant acteurs de la préservation du lac, les institutions environnementales sont paradoxalement à l'origine de problèmes environnementaux d'envergure. C'est notamment le cas de l'Elodée (illustration 10), algue originaire du Canada, introduite par l'INDERENA dans les années 1970 à des fins expérimentales et dont l'expansion, exacerbée par les rejets de gallinaza, est aujourd'hui incontrôlable. En plus d'augmenter fortement le processus d'eutrophisation du lac, l'Elodée a rendu inutilisable près de $12 \%$ de sa superficie, en particulier pour la pisciculture et la navigation (Valderrama, 2013). 
Illustration 10 - L'Elodée, l'une des principales menaces environnementales du lac de Tota

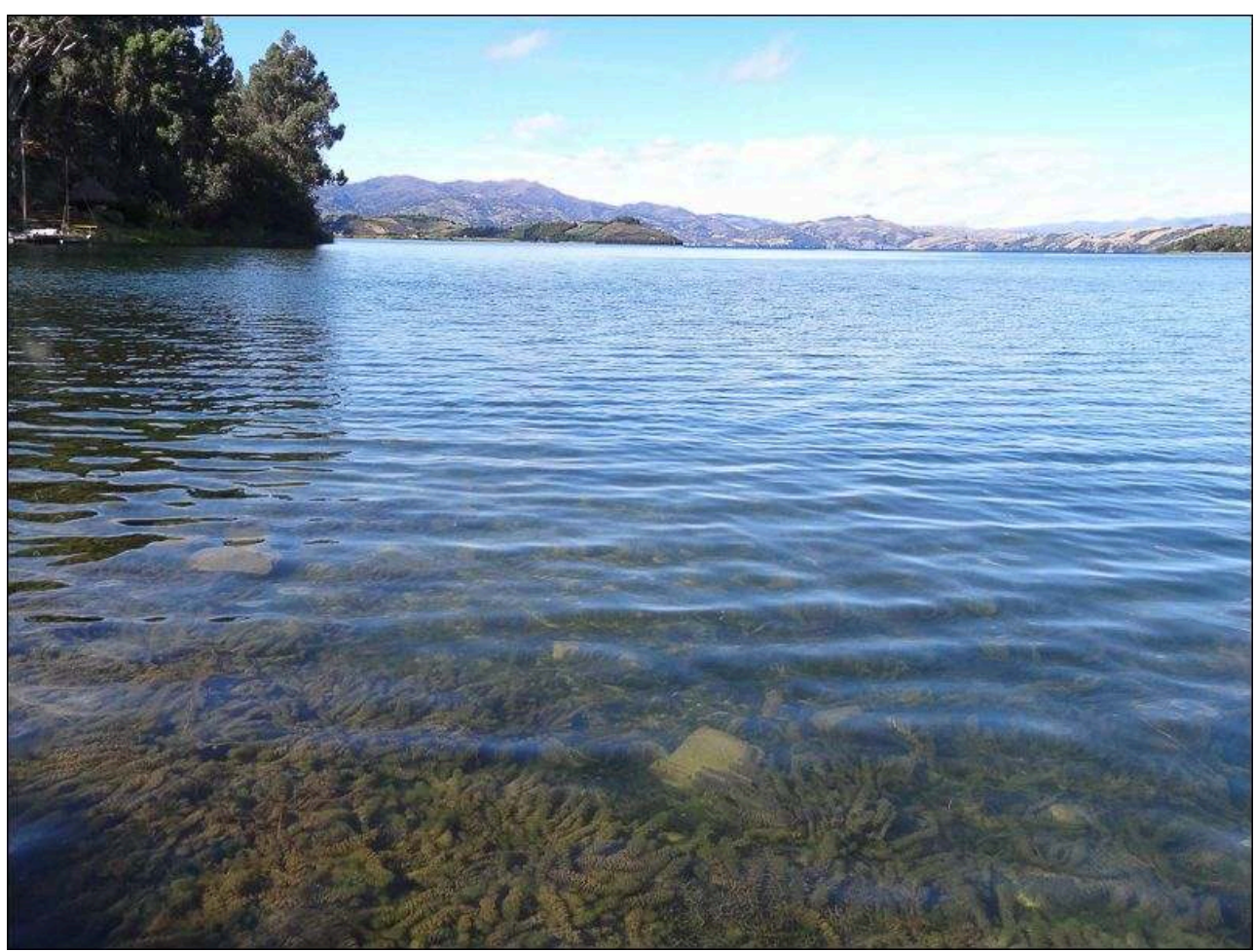

Auteur : D. Leroy, 2014

Biodiversité en déclin, surexploitation de l'eau, contamination des milieux aquatiques, dégradation de l'écosystème, eutrophisation, érosion des sols... le bassin versant du lac de Tota fait l'objet de multiples menaces sur son environnement, qui vont avoir des conséquences considérables sur les relations sociales.

\section{Entre expression des oppositions et prise de parole : le rôle des conflits dans les processus de gouvernance}

Les conflits environnementaux sont avant tout des révélateurs des transformations profondes qui se produisent dans les territoires ainsi que de la difficulté de mise en œuvre de processus de gouvernance impliquant des acteurs obéissant à leur propre logique d'intérêts. S'ils donnent parfois lieu à des rapports de force et des affrontements violents, les conflits ne sont pas toujours néfastes pour la société. Ils peuvent en effet représenter une forme de résistance et d'expression des oppositions, notamment liée à des décisions des pouvoirs publics (Torre, 2015).

\section{Entre les agriculteurs et la CORPOBOYACÁ, des conflits liés à l'appropriation territoriale}

Dans les années 2000, après plusieurs décennies de dégradation environnementale, la préservation du lac de Tota devient un enjeu local, voire national. Animée par de nouvelles préoccupations, la société colombienne aspire en effet à une meilleure prise en charge des atteintes à l'environnement. Chargée de l'administration et de la 
protection du lac, c'est la CORPOBOYACÁ, Corporation autonome régionale, rattachée au Ministère de l'Environnement, qui assure cette fonction. Suite à la résolution $\mathrm{n}^{\circ} 317$ du 29 mars 2007, elle met alors en place un plan d'aménagement POMCA ${ }^{5}$ (CORPOBOYACÁ, 2005).

Parmi les actions mises en application se distingue la conservation du páramo et des cours d'eau qui alimentent le lac de Tota. Afin de limiter la surexploitation de l'eau du lac, la corporation met également en place un système de concessions payantes, sans lesquelles les communautés ne peuvent utiliser l'eau. Plus récemment, en 2012, deux résolutions ( $n^{\circ} 1539$ et $\left.n^{\circ} 1786\right)$ entrent en vigueur afin, d'une part, de contrôler l'augmentation importante des pompes d'eau électriques et thermiques et, d'autre part, de limiter l'avancée des cultures sur les rives du lac. Ces résolutions vont exacerber les tensions avec les communautés, ces dernières exprimant alors leurs désaccords et leur opposition à travers une grande manifestation à Aquitania.

Mais les conflits dans le bassin versant du lac de Tota ne sont pas récents. Déjà en 1975, Perez publiait un ouvrage intitulé Tota, más que un lago es un conflicto ${ }^{6}$. À cette époque déjà, des tensions avaient éclaté entre les communautés rurales, la multinationale sidérurgique Acerías Paz del Río et l'INDERENA au sujet du contrôle du lac. Les conflits sont donc inscrits dans l'histoire de ce territoire et de son aménagement. Marqueurs des évolutions sociales, économiques et environnementales, ils mettent en exergue l'interaction entre les différents acteurs à propos de l'usage de l'espace et de ses conséquences. Synonyme de revendication, les conflits constituent également le moyen d'expression le plus efficace pour s'opposer à des projets d'aménagement qui ne font pas l'unanimité (Torre, 2011).

36 C'est particulièrement le cas du projet d'aménagement POMCA, qui résulte d'une décision gouvernementale de type top-down, sans consultation préalable de la communauté concernée. Or, pour les communautés rurales, qui pratiquent leurs activités agricoles depuis toujours, l'instauration de mesures de restriction est assimilée à un acte de dépossession. Elle génère « un sentiment de privation face à une intervention exogène : l'extérieur vient s'insérer dans des logiques locales » (Lazlaz et Tratnjek, 2011). C'est alors sans surprise que l'acceptation locale de l'institution environnementale ne va pas aller de soi.

"En ce qui concerne le lac de Tota, CORPOBOYACÁ veut plus commander que nous, les propriétaires du lac, parce que pratiquement nous sommes les propriétaires du lac: Tota, Cuítiva et Aquitania, nous sommes les propriétaires directs de ce lac, même s'ils disent que le lac est à l'État, en grande partie, mais il ne peut pas nous interdire de pomper l'eau du lac, comme il l'a fait ici ». (Fernando P., agriculteur, Aquitania, 2015).

Considérant le lac comme leur propriété, les communautés remettent en effet fortement en cause la légitimité de CORPOBOYACÁ à intervenir sur sa gestion, ainsi que les ministères et administrations dont elle dépend. Pour les populations d'Aquitania, de Cuítiva et de Tota, le lac est avant tout pensé comme une ressource territoriale, car il fait partie intégrante des trois municipes. Le lac appartient alors de fait aux riverains. Aussi, contrairement aux populations de l'aval qui ne font que prélever la ressource, les municipes d'Aquitania, de Cuítiva et Tota font partie des bassins hydrographiques qui alimentent ce grand réservoir naturel. La notion de bassin versant justifie donc le droit de propriété. "On alimente le lac donc on a le droit de l'exploiter». Pour les populations locales, la question de la localisation dans l'espace leur donne ainsi une légitimité pour utiliser l'eau du lac à leur guise. Elles se considèrent comme les propriétaires légitimes, et critiquent fortement la consommation de l'aval, en 
particulier celle destinée à des fins industrielles (Acerías Paz del Río). Il est d'ailleurs intéressant de souligner que pour beaucoup d'agriculteurs, l'irrigation est considérée comme la pratique la plus durable, car l'eau revient toujours au lac par gravité, contrairement à la consommation aval. À la légitimation par la situation dans l'espace bassin versant vient se superposer la légitimation par le temps : les populations locales sont installées dans ces hautes terres depuis des temps immémoriaux. Elles s'arrogent donc le droit de décider des aménagements du bassin versant. On observe ainsi une démonstration de "légitimation par l'espace et par le temps " (Lazlaz et Tratnjek, 2011).

Pour la corporation autonome, le lac, tout comme l'ensemble des ressources naturelles du territoire colombien, appartiennent à l'État, comme l'indique le décret 2811 de 1974 $\mathrm{du}$ code national des ressources naturelles renouvelables et de protection de l'environnement. À l'inverse des agriculteurs, CORPOBOYACÁ revendique l'utilisation de la ressource par les populations de l'aval. En tant qu'autorité environnementale, la préservation est aussi au centre du discours des membres de la corporation.

"Selon la loi, les ressources naturelles appartiennent à l'État, c'est l'État qui est chargé de l'administration, mais [les agriculteurs] croient que ça leur appartient, ils n'ont pas changé cette mentalité de "c'est à moi", "le lac ne va jamais se sécher » ou encore "moi je suis né ici et le lac est à moi ". Ils n'ont pas cette perspective; et ceux qui sont en aval du bassin versant revendiquent également le droit du lac, et ceux de l'amont que "le lac est à eux». C'est pour ça que CORPOBOYACÁ existe, pour l'administration des ressources, pour la préservation, veiller à la préservation ». (Directeur du centre CORPOBOYACÁ de Santa Inès, 2015).

Mais chez les agriculteurs, on dénonce au contraire les pratiques de la CORPOBOYACÁ, qui serait à l'origine de tous les maux du lac, notamment ceux liés à l'invasion de l'Elodée. En plus d'avoir rendu inutilisable une grande partie du lac et participé au processus d'eutrophisation, la corporation est souvent accusée d'« être plus préoccupée par les concessions que par les contrôles ", car mue par une volonté d'enrichissement économique. Au regard des entretiens, les agriculteurs se considèrent davantage comme des victimes, de parfaits boucs émissaires, pointés par la corporation comme responsables des problèmes d'environnement. Pour leur défense, ils citent fréquemment d'autres sources de pollution et de surexploitation de l'eau que l'agriculture, telles que les activités urbaines et industrielles.

\footnotetext{
"Ils ont trouvé l'agriculteur pour l'accuser de la pollution du lac, l'agriculteur, uniquement, que nous sommes en train de détériorer le lac, que nous sommes en train de baisser son niveau, que nous sommes en train d'utiliser l'eau pour l'irrigation. Mais ils ne parlent jamais de l'eau qu'ils prennent pour l'industrie, de l'eau qu'ils prennent pour les autres municipes. Les fonds qu'ils donnent à cette corporation sont seulement utilisés pour la bureaucratie!» (Carlos P., agriculteur, Aquitania, 2015).
}

La légitimité de la corporation est donc au plus bas chez les communautés rurales, qui revendiquent une responsabilité partagée, voire une externalisation de responsabilité. Aussi, l'autorité CORPOBOYACÁ est ici vécue comme un rapport de domination sociale, où les élites politiques tentent d'imposer des restrictions d'usages aux communautés qui possèdent pourtant un savoir pratique de l'environnement du lac.

"Ils sont ignorants, ils ne savent rien, ils n'ont aucune personne compétente. Ce sont des postes politiques qui ne servent à rien, on sait plus nous, on sait beaucoup plus de la gestion et on a davantage conscience de préserver le lac parce qu'on vit du lac et eux ils sont seulement ici pour des postes politiques. Ils sont une autorité environnementale, mais ils n'ont pas de conscience environnementale. Ce qui les intéresse ce sont les résultats 
économiques. Ils ont un lac totalement abandonné ». (Hugo M., agriculteur, Aquitania, 2015).

\section{De la médiatisation des problèmes environnementaux à la gouvernance multi-niveaux : le rôle du Globo gris}

41 Du côté de la société civile, la place occupée par la CORPOBOYACÁ dans la gestion des problèmes d'environnement fait également l'objet de nombreuses critiques. L'idée qu'une autorité unique soit légitime pour gérer seule les contraintes environnementales autour du lac est en effet fortement remise en cause. C'est pourquoi plusieurs acteurs issus de la société civile vont décider de s'impliquer dans les processus de décision afin de participer à la gouvernance du lac.

"CORPOBOYACÁ a reçu un cadeau: le lac de Tota, mais elle l'a sous-estimé, après la formulation du POMCA, absolument rien n'a été fait pour administrer ce bien public. Maintenant en tant que citoyens, nous avons aussi la responsabilité de participer à cette administration du lac et c'est pour ça qu'il existe des mécanismes de participation citoyenne mise en place par la constitution de 91 ». (Biologiste, professeur Universidad Pedagógica y Tecnológica de Colombia, cité par Orozco Arcila et al., 2012).

Parmi les initiatives de la société civile se détachent les actions d'une association, la fondation Montecito. En 2011, cette dernière donne naissance à la Causa Tota à travers une pétition adressée au gouvernement. L'objectif de cette cause est d'œuvrer pour la " récupération et la préservation perpétuelle du lac de Tota et de son bassin versant ". En quelques semaines, elle se matérialise par la création d'un mouvement civique : $E l$ Mocilato (movimiento cívico pro lago de Tota y su cuenca ${ }^{7}$ ).

L'une des actions phares de la Causa Tota est sans conteste le dénudement collectif de manifestants organisé en 2012 sur les rives du lac afin d'attirer l'attention nationale sur la problématique de l'écosystème (illustration 11). Mais le tournant de la Causa Tota va avoir lieu quelques mois plus tard avec l'obtention du Globo gris, prix décerné par la WWN (World Wetland Network ou réseau mondial des zones humides) pour signaler les menaces environnementales qui pèsent sur des zones humides d'importance internationale. La campagne pour l'obtention du prix a été menée par l'association environnementale Montecito, à travers un processus de vote sur le site web de la WWN. Si l'objectif de l'association était d'attirer l'attention nationale et internationale sur la situation actuelle $d u$ lac, l'obtention du prix va être à l'origine de tensions particulièrement vives entre les différents acteurs du lac et le directeur de la fondation Montecito, Felipe Velasco. 
Illustration 11 - Dénudement collectif sur les rives du lac de Tota

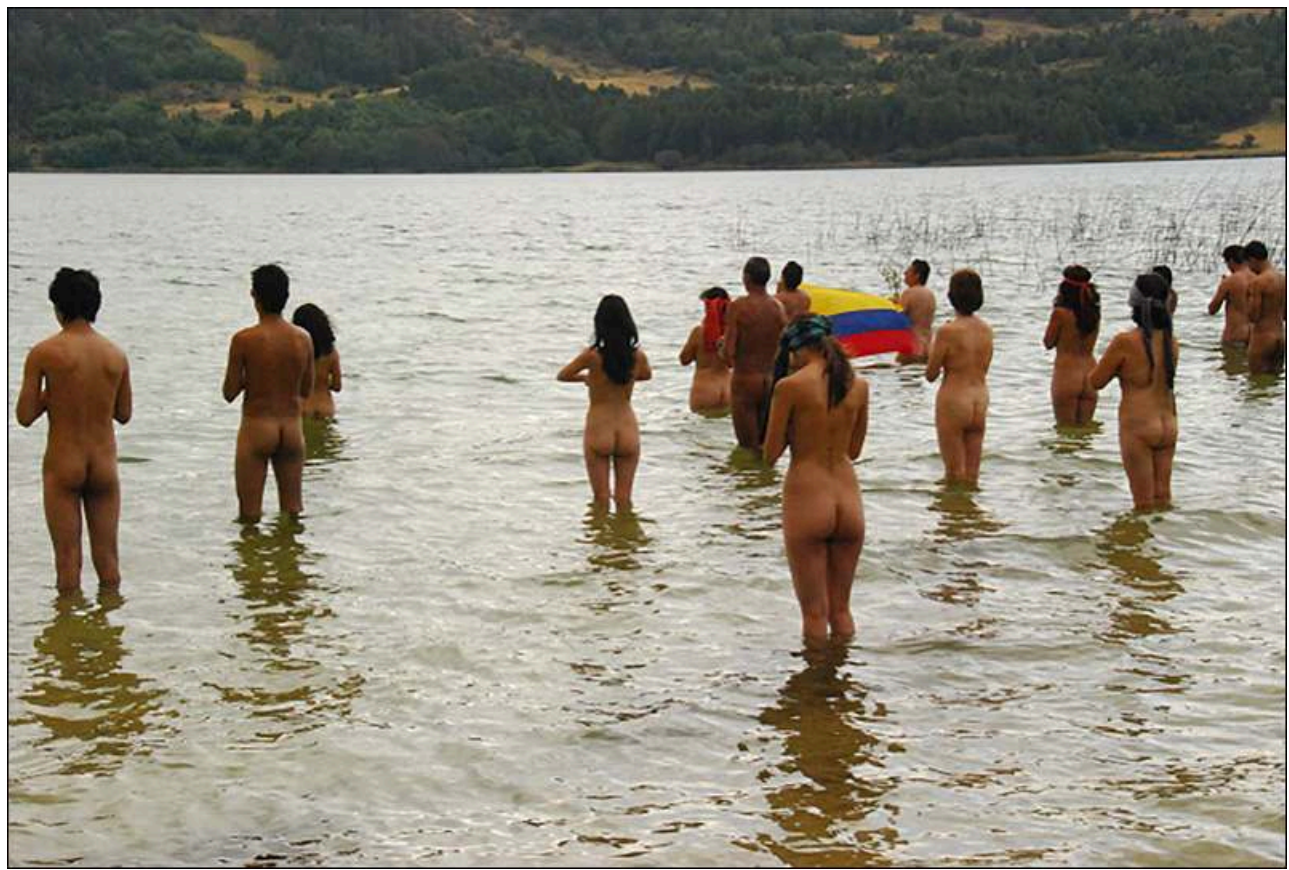

Auteur : José Lizarazo, 2012. nationaux et internationaux. On ne parle plus seulement de menaces environnementales, mais « du lac le plus pollué au monde », d'《 un égout ». L'impact sur les principales activités économiques du lac va alors être considérable. La truite de Tota et la ciboule d'Aquitania ne se vendent plus aussi bien sur principaux marchés du pays. Les réservations hôtelières diminuent fortement. Le lac de Tota acquiert alors une connotation négative pour de nombreux Colombiens.

«Ils ont stigmatisé la région, le lac le plus pollué du monde? Non, ce n'est pas le lac le plus pollué du monde !» (Directeur du centre de CORPOBOYACÁ de Santa Inès, 2015).

L'impact de cette médiatisation n'est d'ailleurs pas seulement économique. Il est aussi social. Car étant dénoncé par les médias comme les principaux coupables de la dégradation du lac, les communautés rurales se sentent totalement stigmatisées : «On dit que c'est nous les agriculteurs qui polluons le lac, qu'on est en train de le faire disparaitre ». Pour l'ensemble des paysans, mais aussi des pisciculteurs, Felipe Velasco est le seul responsable de cette crise. Il cristallise toute la haine de la population d'Aquitania, jusqu'à être victime de menaces.

"Les marchés l'ont ressenti et l'homme n'est pas très aimé à Aquitania, pas du tout même. Il n'a pas mesuré les conséquences de ce qu'il disait [...] Il aurait pu provoquer un conflit social sans précédent. Imagine 4000 personnes sans emploi... » (Lucio T., agriculteur, Aquitania, 2014).

Pour les autorités environnementales et les institutions locales, l'attribution du Globo gris dérange également, car elles se voient totalement décrédibilisées. C'est notamment le cas de la mairie d'Aquitania et de la CORPOBOYACÁ qui entretiennent des relations très tendues avec l'association Montecito. Mais malgré les tensions qu'il génère, le prix Globo gris constitue un coup de projecteur sans précédent sur l'état de dégradation du lac de Tota. Il fait d'ailleurs les titres de plusieurs journaux nationaux et internationaux tels que El Tiempo, El Espectador et BBC monde. Si bien que les politiciens de Boyacá 
engagent un débat au Congrès colombien pour discuter de l'enjeu du plus grand lac de la nation. Au-delà des tensions qu'il génère, le Globo gris va donc entraîner une période d'avancée et d'innovation.

En effet, en 2013, l'Agence française pour le développement (AFD) et le Ministère de l'Environnement de Colombie (MADS) mettent en place une coopération technique afin de favoriser la préservation de cet écosystème unique au monde. Cette coopération s'est essentiellement appuyée sur un don de 3 millions d'euros. En 2014, fut également approuvé le document CONPES 3801 intitulé Manejo Ambiental Integral de la Cuenca Hidrográfica del Lago de Tota ${ }^{8}$ dont l'objectif est de promouvoir le développement intégré et durable du bassin versant. Il s'agit plus particulièrement d'un plan d'actions dont $49 \%$ seront financés par l'État et le reste par les acteurs régionaux et locaux (DNP, 2014). À cette même époque se mettent aussi en place deux instances de coordination impliquant les différents acteurs du bassin versant du lac de Tota : la Mesa de trabajo Permanente del Lago de Tota ${ }^{9}$ (2013) et le Consejo de Cuenca del Lago de Tota ${ }^{10}(2014)$, instances que nous détaillerons par la suite. Enfin le 29 avril 2015, la cour constitutionnelle de Colombie a déclaré inapplicable le décret 1111 de 1952 qui permettait l'utilisation exclusive et illimitée dans le temps des eaux du lac de Tota par l'entreprise sidérurgique Acerías Paz del Río. Ce qui montre une véritable volonté d'action.

La gouvernance du bassin versant du lac de Tota devient donc multi-niveaux (Bache et Flinders, cité par Torre, 2015), avec les politiques publiques nationales et des aides internationales. Mais elle prend également sa source à un niveau plus local, à travers des instruments concrets de gestion de l'environnement (CONPES 3801) et la mise en place d'instances de coordination. La médiatisation du Globo gris a donc entraîné des changements profonds de la gestion des problèmes environnementaux autour du lac. Elle a permis de nouveaux accords au niveau local, national, voire international, mais aussi de nouvelles négociations des actes techniques, avec une révision des plans d'aménagements (POMCA).

"En montrant la réalité, l'objectif est qu'il y ait une réaction positive de tous. Une réaction des citoyens, du gouvernement, internationale aussi, des ONG. Finalement, c'est mettre un coup de projecteur. De fait, grâce au Globo gris on a obtenu [les aides] de la France, grâce à ce Globo gris est apparu le CONPES aussi, l'argent de la Colombie, le Consejo de Cuenca. Tout ça a été une réaction en chaine, après le Globo gris. Beaucoup de choses se sont passées après le Globo gris. Mais le moment du Globo gris est un moment très désagréable. Parce que c'est une information qui met mal à l'aise. On a alors reçu beaucoup de critiques en 2012, surtout de CORPOBOYACÁ parce que ça les gênait qu'on montre la vérité... ». (Felipe Velasco. Directeur de la Fondation Montecito, Sogamoso, 2015).

Or, bien qu'elle ait permis à la société de reconsidérer son territoire, l'attribution du Globo gris a également généré des conflits entre les acteurs, faisant du lac de Tota un espace de tensions. À cette époque, les manifestions, les débats et les oppositions n'ont en effet pas été rares. Mais bien que ces conflits aient donné lieu à des rapports de force, ils ont été un mal nécessaire pour attirer l'attention nationale et internationale sur l'état de dégradation du bassin versant du lac de Tota. Les conflits ont donc constitué une plateforme de prise de parole, une forme d'expression des désaccords, contribuant à la prise en compte des problèmes environnementaux dans les politiques d'aménagement. Ils ont également été à l'origine de nouvelles formes de coopération entre les acteurs. Finalement c'est l'ensemble des relations sociales qui ont été transformées dans cet espace en mutation. C'est pourquoi il est essentiel de s'intéresser 
aux différents types de liens qui unissent les acteurs impliqués dans la gouvernance du lac de Tota.

\section{L'étude des réseaux sociaux : clé de compréhension des conflits et des modes de coopération}

Selon Cadoret (2006), les réseaux sociaux représentent un outil de compréhension des conflits d'usage et des modes de coopération. Ils évoquent l'ensemble des acteurs (dans notre cas les agriculteurs, institutions, associations...) et les relations qu'ils entretiennent entre eux (hiérarchique, conflictuelle, de coopération...). D’abord utilisée en sociologie, cette notion est aujourd'hui mobilisée par de nombreux géographes (Balki, 1993 ; Offner et Pumain, 1996 ; Cadoret, 2006), notamment pour appréhender les interactions entre une grande diversité d'acteurs dans une perspective spatiale.

Il existe aujourd'hui plusieurs outils d'analyse des réseaux sociaux (systèmes multiagents, analyses structurales...). Néanmoins, l'analyse des réseaux sociaux par la systémique et les graphes semble la plus pertinente pour comprendre comment les rapports des acteurs s'articulent à différentes échelles spatiales (Cadoret, 2006).

Le graphe ci-dessous (illustration 12), largement inspiré des travaux de Cadoret (2006), représente les liens entre les principaux acteurs impliqués dans la gouvernance du lac de Tota. Les flèches en noir montrent les relations de hiérarchie entre l'État, le conseil régional et les mairies, mais aussi entre le Ministère de l'Environnement et la CORPOBOYACÁ. Ces relations d'autorités sont centrales notamment pour la gestion du CONPES, dont $49 \%$ du financement proviennent de l'État.

Illustration 12 - Graphe des réseaux d'acteurs impliqués dans la gouvernance du bassin versant du lac de Tota

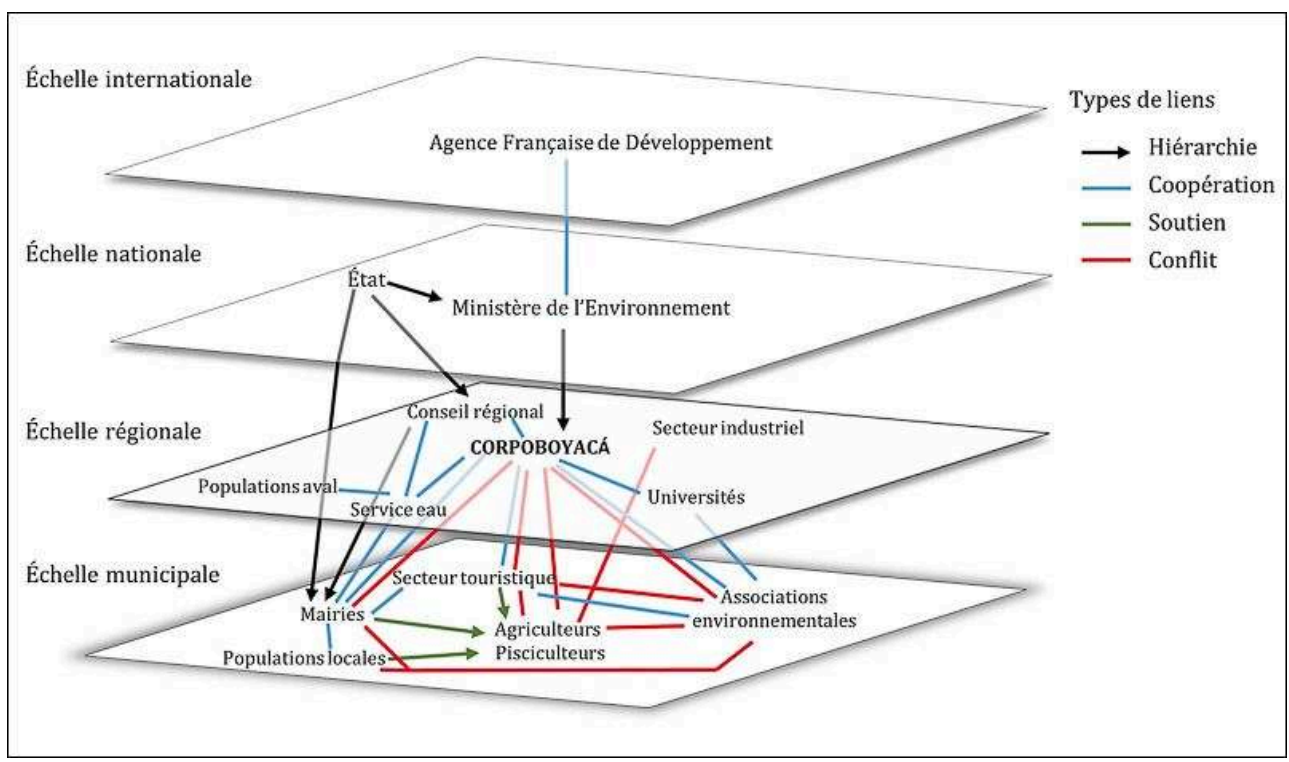

Auteur : D. Leroy, 2017

Les liens de coopération se matérialisent par des traits bleus. Ils montrent notamment la relation entre les universités et la CORPOBOYACÁ : le POMCA, plan d'aménagement, a par exemple été établi à travers une convention avec l'Université Javeriana, mais aussi 
entre l'Agence Française de Développement et le Ministère de l'Environnement colombien. $\mathrm{du}$ lac de Tota. Ces relations conflictuelles sont particulièrement vives entre les agriculteurs, la CORPOBOYACÁ et les associations environnementales (en particulier la Fondation Montecito). Toutefois, le conflit n'est pas seulement un élément négatif. Il crée des relations de solidarité entre certains acteurs, comme en témoignent les liens de soutien des populations et des mairies locales à l'égard des agriculteurs et des pisciculteurs. étroites entre les conflits et les processus de coopération. Par exemple, les mairies vont à la fois être en conflit avec la CORPOBOYACÁ, car elles soutiennent les agriculteurs, mais aussi en partenariat avec cette corporation autonome pour la préservation du lac. Ces liens doubles sont également manifestes entre les associations environnementales, le secteur touristique et la CORPOBOYACÁ, ce qui prouve que les conflits accompagnent les processus de coordination.

Finalement, l'ensemble de ces relations, qui s'articulent à différentes échelles spatiales, démontre que la gouvernance du bassin versant est multi-niveaux, mais aussi, et surtout, un enjeu de pouvoir.

\section{La gouvernance territoriale de l'environnement : enjeux de pouvoirs}

\section{Des instruments financiers sources de tensions}

Malgré la période d'avancée et d'innovation provoquée par Globo gris, la gestion des instruments financiers octroyés pour la préservation du lac va être marquée par des tensions. En effet, deux ans après le don de l'AFD au Ministère de l'Environnement colombien, plusieurs acteurs (associations, agriculteurs, secteur touristique...) s'interrogent sur l'utilisation de ces financements.

«Je ne sais pas si c'est la France ou un autre pays qui a donné 40 millions pour le lac de Tota, pour le préserver. Moi, je peux vous dire qu'ils n'ont rien fait au lac de Tota [...] il y a une corruption hallucinante en Colombie, parce que les mêmes employés de l'État, ceux qui gèrent, volent tout l'argent et ne font rien ». (Alfonso C., agriculteur. Aquitania, 2015).

Selon la CORPOBOYACÁ, qui est chargée d'administrer les aides financières de l'AFD, le retard des investissements est dû à la lenteur administrative du Ministère de l'Environnement, qui est censé distribuer l'argent à travers plusieurs conventions. Mais pour certains acteurs du bassin versant, les soupçons de corruption sont grands. La gestion des aides externes (AFD) et des investissements publics (CONPES) devient alors un enjeu central pour les acteurs privés et issus de la société civile. Et la participation aux instances de coordination un moyen déterminant pour prendre part aux processus d'administration :

"Le lac de Tota il faut le protéger, et pour le protéger il faut des ressources. Aujourd'hui, le gouvernement a fourni un instrument qui s'appelle CONPES. C'est un budget spécifiquement destiné à un thème, dans ce cas, le lac de Tota. Et ce budget il faut l'utiliser de la meilleure façon. Le Consejo de Cuenca n'est rien d'autre que l'administration, comme si on faisait la comparaison avec l'administration d'un immeuble. Ceux qui vont participer, ou qui aspirent 
à participer à cela, c'est pour veiller qu'ils ne volent pas les ressources. Qu'ils ne les utilisent pas pour des choses qui n'ont rien à voir ». (Oscar R., Directeur de la Reserva Natural Pueblito Antiguo (acteur du tourisme), Aquitania, 2014).

\section{La " Mesa de trabajo Permanente » et le "Consejo de Cuenca » : deux instances de coordination parallèles et concurrentes} place d'une formation en résolution des conflits, la Mesa de trabajo Permanente n'apporte pas de réelle contribution à la préservation du lac. De plus, elle est toujours assimilée à une "volonté de la Corporation pour maintenir et dominer la gouvernance ${ }^{12}$. Les suspicions de corruption sont d'ailleurs fortement dénoncées. Et le dialogue avec les communautés rurales reste très tendu. De ce fait, de vives tensions éclatent en décembre 2014 entre les agriculteurs et la CORPOBOYACÁ suite à l'élaboration d'une nouvelle mesure visant à restreindre l'irrigation de jour. Mais avant même son approbation, cette mesure est à l'origine d'une vague de contestation sans précédent à Aquitania, ce qui montre que les conflits restent encore le moyen privilégié pour les communautés d'infléchir les décisions, au détriment de la participation à la Mesa Permanente. différents acteurs (socio-économiques, société civile, pouvoirs publics...) de participer aux processus de décisions. Elles peuvent être considérées comme des espaces de concertation permettant de faire des choix collectifs. Leur mode de fonctionnement détermine donc fortement les formes de gouvernance.

coordination mise en place par le Ministère de l'Environnement ${ }^{11}$ en mai 2013, afin « de veiller à la récupération, défense, protection, conservation et utilisation durable du bassin versant du lac de Tota" (Ministerio de Ambiente et CORPOBOYACÁ, 2013). Administré par la CORPOBOYACÁ, son objectif est de générer un espace de participation à des processus de décision de l'ensemble des acteurs privés et publics. Toutefois, la place de l'État, et plus particulièrement de la CORPOBOYACÁ, est centrale, puisque c'est elle qui propose, voire impose les procédures. Bien que cette instance soit ouverte à une diversité de parties prenantes, elle laisse peu de place aux décisions endogènes. $L a$ Mesa de trabajo Permanente peut donc être considérée comme une instance de coordination relevant d'une gouvernance publique.

par certains acteurs, notamment la fondation Montecito. Car selon Felipe Velasco, qui est « exclu» du dialogue, la Mesa de trabajo Permanente n'offre pas une participation réelle et effective à l'ensemble des acteurs. Il n'existe en effet qu'un seul représentant pour chaque secteur (ex: ONG, associations...). De plus, les absences de certains représentants sont très fréquentes, les quorums et les actes publics inexistants et les objectifs, essentiellement orientés autour de la formation, sont très loin du processus de prise de décision recherché. Si bien que le directeur général de CORPOBOYACÁ se dit "profondément préoccupé » par les résultats de la Mesa de trabajo Permanente. D’autant plus que les conflits sont récurrents.

Malgré une volonté affichée d'instaurer un espace de dialogue, à travers la mise en Face à l'émergence de nouvelles demandes sociales en termes de gouvernance de l'environnement, la société civile sollicite en novembre 2014, la mise en place du Consejo 
de Cuenca, instance consultative et représentative de tous les acteurs qui vivent et développent des activités à l'intérieur d'un bassin versant (MADS, 2013). Dans la lignée de la Mesa de trabajo Permanente, l'objectif du Consejo de Cuenca est de «contribuer à l'existence d'une utilisation rationnelle et durable du lac de Tota et de son bassin versant, en procurant une amélioration de ses caractéristiques écologiques et du bienêtre des communautés " (CCLT, 2015). Il s'agit également d'une figure de loi suite au décret 1640 de 2012 (aujourd'hui décret 1076 de 2015) du Ministère de l'Environnement. C'est donc à la charge de l'État, et plus particulièrement de la CORPOBOYACÁ, de mobiliser les acteurs associatifs, privés et publics autour du territoire, même si l'initiative reste citoyenne. Toutefois, venant directement concurrencer la Mesa de trabajo Permanente, la mise en place du Consejo de Cuenca n'est pas allée de soi. Car cette instance de coordination est essentiellement réservée aux acteurs endogènes. La CORPOBOYACÁ ainsi que les autres institutions étatiques sont par exemple exclues. Le Consejo de Cuenca peut donc être considéré comme une instance de coordination relevant d'une gouvernance citoyenne (illustration 13).

"Les seules institutions gouvernementales qui font partie du Consejo de Cuenca sont les mairies, rien de plus. C'est un aspect important parce que ça donne au Consejo de Cuenca une indépendance de l'autorité environnementale. On est alors comme une autorité, mais essentiellement de la société civile». (Felipe Velasco. Directeur de la Fondation Montecito, Sogamoso,2015).

Illustration 13 - Principaux acteurs de la Mesa de trabajo Permanente et du Consejo de Cuenca du lac de Tota en 2015

\begin{tabular}{|c|c|c|}
\hline & \multicolumn{2}{|l|}{ Instances de coordinations } \\
\hline & $\begin{array}{l}\text { Mesa de trabajo } \\
\text { Permanente } \\
\text { Gouvernance publique }\end{array}$ & $\begin{array}{l}\text { Consejo de Cuenca } \\
\text { Gouvernance citoyenne }\end{array}$ \\
\hline & \multicolumn{2}{|l|}{ Administration } \\
\hline & CORPOBOYACÁ & Société civile \\
\hline & \multicolumn{2}{|l|}{ Principaux acteurs } \\
\hline \multirow{3}{*}{ National } & Ministerio de Ambiente & \\
\hline & Ministerio de Agricultura & \\
\hline & Planeación Nacional & \\
\hline \multirow{3}{*}{ Régional } & Procuraduría Regional & \\
\hline & Gobernación de Boyacá & \\
\hline & CORPOBOYACÁ & \\
\hline \multirow[t]{2}{*}{ Local } & \multicolumn{2}{|c|}{ Mairie d'Aquitania, Cuítiva, Tota } \\
\hline & Juntas de acción comunale & \\
\hline
\end{tabular}




\begin{tabular}{|l|l|}
\hline \multicolumn{2}{|l|}{ Agriculteurs : associations de producteurs, associations d'irrigants } \\
\hline Pisciculteurs \\
\hline Secteur touristique \\
\hline $\begin{array}{l}\text { Recherche et éducation : Universitad de Boyacá, Universidad Pedagógica y Tecnológica } \\
\text { de Colombia, Universidad Javeriana... }\end{array}$ \\
\hline \multirow{3}{*}{ Un siège pour les ONG } & Fundación Montecito \\
\hline \multirow{5}{*}{ ONG Tejido Ambiente } \\
\hline \multirow{3}{*}{$\begin{array}{l}\text { ONG Fundación Amigos Pro Desarrollo municipio de Cuítiva y } \\
\text { Defensa del Lago de Tota }\end{array}$} \\
\cline { 2 - 2 } & Futuro Verde \\
\cline { 2 - 2 } & ONG Ambientalista Lago de Tota \\
\hline
\end{tabular}

est précisément du rapport de pouvoir entre les deux instances de coordination que dépend la gestion des finances et des aides financières étrangères. C'est pourquoi la CORPOBOYACÁ a pendant longtemps opposé une certaine résistance à l'organisation du Consejo de Cuenca. Toutefois, face à la pression de la société civile, l'instance de coordination a finalement vu le jour, alors que la Mesa de trabajo Permanente s'est provisoirement effacée. octobre 2016. Selon la CORPOBOYACÁ, « l'objectif est de faire que les communautés en général se donnent les moyens et travaillent collectivement pour les bénéfices du lac ». Mais pour Felipe Velasco, la réactivation de la Mesa de trabajo Permanente n'est finalement qu'un moyen pour reprendre le contrôle de la gouvernance.

«Il y a de graves conflits d'intérêts. La réactivation de la MPLT (Mesa de trabajo Permanente del Lago de Tota) est aussi la conséquence du manquement du CCLT (Consejo de Cuenca del Lago de Tota) à son devoir. Le premier point ne serait jamais arrivé sans le second. Maintenant, avec les deux sur la table, il y a une grande confusion et désinformation, ce qui représente un scénario idéal pour que règne la volonté de l'autorité environnementale (CORPOBOYACÁ), et séteigne la gouvernance. Lamentable ». (Felipe Velasco. Directeur de la Fondation Montecito, 2017).

Aujourd'hui, en 2017, les deux instances de coordination, bien qu'étant concurrentes, cohabitent au sein du bassin versant du lac de Tota. CORPOBOYACÁ est par exemple 
venu intégrer le Consejo de Cuenca, alors qu'un représentant du Consejo de Cuenca participe aux réunions de la Mesa de trabajo Permanente. Les objectifs des deux instances sont aussi plus clairs. La Mesa de trabajo Permanente veille à la réalisation des investissements économiques (CONPES et AFD), tandis que les actions du Consejo de Cuenca se concentrent essentiellement sur la participation dans l'actualisation du projet d'aménagement (POMCA). Cela devrait permettre à certains acteurs qui n'avaient pas été consultés dans les phases antérieures d'élaboration des projets (en particulier les communautés rurales) d'influencer les décisions. Toutefois, les conflits d'intérêt sont toujours latents entre les deux instances, notamment liés à l'exercice du pouvoir. De plus, la démission récente (en mars 2017) de Felipe Velasco du CCLT interroge sur l'efficacité réelle de cette instance de coordination, qui semble avoir perdu son indépendance depuis l'intégration de la CORPOBOYACÁ.

Nous proposons en illustration 14 un schéma récapitulatif afin de mettre en lumière les processus de gouvernance et de conflits d'usage au sein du bassin versant du lac de Tota. Les premiers conflits sont apparus dans les années 1960. Ils opposaient essentiellement les communautés rurales et les institutions environnementales. À partir des années 2000, ces conflits se sont intensifiés, à la mise en place d'un plan d'aménagement impliquant des restrictions d'usage pour les communautés rurales. Mais c'est surtout dans les années 2010 que les situations conflictuelles sont devenues particulièrement vives, notamment suite à la médiatisation des problèmes environnementaux (globo gris) par une association environnementale (Montecito). Expression des désaccords, ces conflits ont permis une prise en compte des questions environnementales dans les politiques de développement du territoire, contribuant à l'émergence de nouvelles formes de coordination entre les acteurs. Toutefois, la création de deux structures de gouvernance (Mesa de trabajo Permanente et Consejo de Cuenca), parallèles et concurrentes, montre que la fin des conflits n'est pas pour demain.

Illustration 14 - Processus de gouvernance et conflits d'usage au sein du bassin versant du lac de Tota

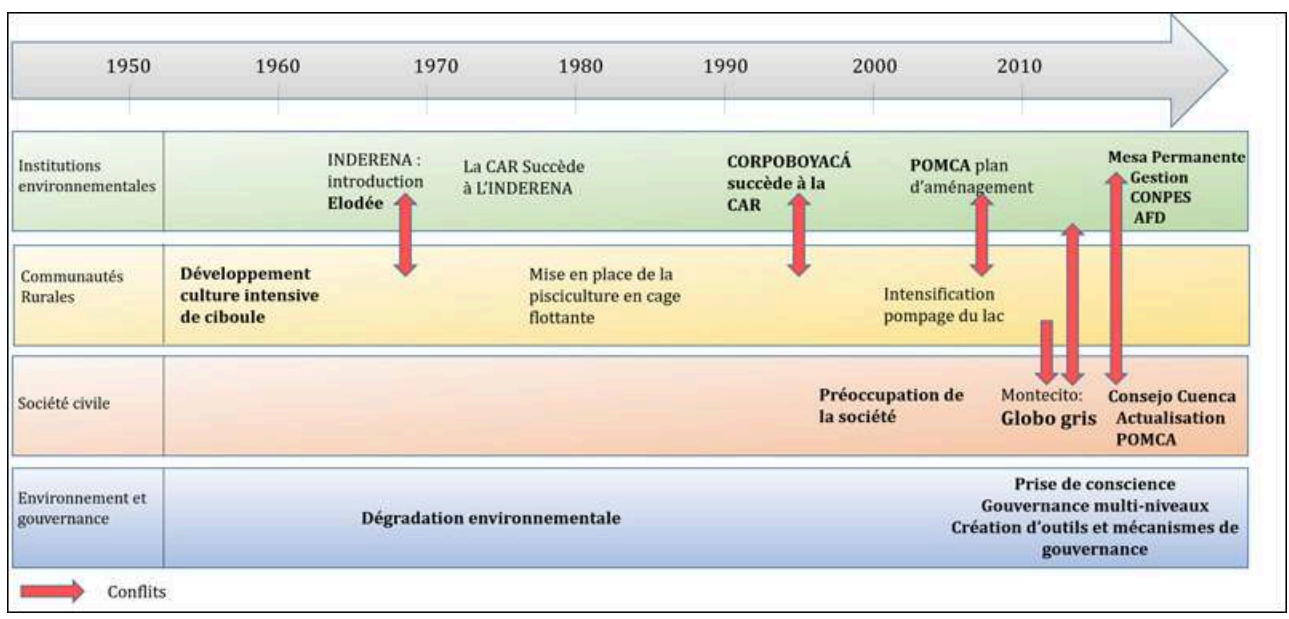

Auteur : D. Leroy, 2017 


\section{Conclusion}

Tota est aujourd'hui un enjeu local, national, voire international. Longtemps négligé, l'environnement est devenu un élément central des processus de gouvernance du territoire, témoignant de l'aspiration sociale d'une nouvelle forme de gestion, ainsi que la demande d'outils adaptés, au service de la protection du plus grand lac de Colombie. Toutefois, la prise en compte des problèmes environnementaux par les différents acteurs impliqués dans la gouvernance du bassin versant du lac est encore loin d'aller de soi, puisque les conflits sont omniprésents.

Néanmoins, il serait sans doute réducteur de voir le conflit comme un fait seulement négatif, un dysfonctionnement qu'il s'agit d'éliminer au plus vite. Car sous un autre angle, le conflit peut être considéré comme une modalité de coordination des acteurs, un facteur d'innovation. Révélateur des transformations sociales, économiques et environnementales, les conflits ont en effet largement contribué à l'expression des oppositions des communautés rurales aux nouvelles restrictions d'usage imposées par CORPOBOYACÁ. Parallèlement, les conflits ont constitué des plateformes de prise de parole pour des catégories d'acteurs négligées ou oubliées dans les structures de gouvernance (Torre, 2011a), comme ce fut le cas de la société civile, qui, à travers le Globo gris, a contribué à la prise en compte des problèmes environnementaux dans les politiques d'aménagement. Loin d'être seulement un élément négatif, les conflits participent donc aux processus de gouvernance territoriale de l'environnement.

Cependant, en tant qu'enjeu de pouvoir, la gouvernance continue aussi d'être une source d'oppositions, de tensions. En témoigne la concurrence entre les deux instances de coordination qui met en lumière les rapports de pouvoir entre les pouvoirs publics et la société civile.

L'intégration du bassin versant du lac de Tota dans la convention de RAMSAR (Convention relative aux zones humides d'importance internationale particulièrement comme habitats des oiseaux d'eau) est en attente depuis 2012. Si elle est validée, elle pourrait impulser une nouvelle dynamique de gouvernance de l'environnement sur ce territoire. Mais en raison des restrictions d'usage qu'elle implique, la convention de RAMSAR attise déjà les tensions chez les communautés rurales. La fin des conflits n'est donc pas à l'ordre du jour au sein du bassin versant du lac de Tota.

\section{BIBLIOGRAPHY}

Bakis H., 1993. Les réseaux et leurs enjeux sociaux. Paris PUF, Que Sais-je?, 128 p.

Beaurain C., 2002. Gouvernance environnementale locale et comportements économiques. Un nouvel éclairage sur l'articulation du temps et de l'espace. Développement durable et territoires, Dossier 2, http://developpementdurable.revues.org/1110 ; DOI : 10.4000/developpementdurable. 1110

EchoGéo, 43 | 2018 
Beuret J. E., Cadoret A., 2011. Une gouvernance territoriale endogène de l'environnement : contours et enjeux. Géographie, économie, société, vol. 13, n 4, p. 363-386.

Cadoret A, 2006. Conflits d'usage liés à l'environnement et réseaux sociaux : enjeux d'une gestion intégrée ? Le cas du littoral du Languedoc-Roussillon. Thèse de doctorat, Université Paul ValéryMontpellier III.

Chaparro Valderrama J., 2013. Cambios institucionales para preservar la cantidad y la calidad del agua en la cuenca del Lago de Tota. Thèse de maîtrise, Pontificia Universidad de Javeriana, Bogota.

Corpoboyacá, 2005. Documento Síntesis POMCA. Plan de Ordenación y Manejo de la Cuenca del Lago de Tota.

Consejo de Cuenca del Lago de Tota, 2015. Reglamento interno Consejo de Cuenca del Lago de Tota. 8 p. Decreto 2811 de 1974. Código Nacional de Recursos Naturales Renovables y de Protección al Medio Ambiente. Bogotá, 1974.

Departamento Nacional de Planeación DNP, 2014. Conpes 3801, Manejo Integral de la cuenca hidrográfica del lago de Tota.

Froger G., 2007. Environnement et gouvernance : de la politique publique à la régulation de l'action collective. Jeux de gouvernance. Regards et réflexions sur un concept. In Hufty M., 2007, Jeux de gouvernance : Regards et réflexions sur un concept. Karthala, p. 29-51.

Hinnewinkel C., Guillerme S., 2006. Contraintes environnementales et gouvernance des territoires. Natures Sciences Sociétés, Compte rendu de colloque (Lille, 23-24 septembre 2004), vol. 14, p. 99-101. http://www.cairn.info/revue-natures-sciences-societes-2006-1-page-99.htm Ghiotti S., 2006. Les Territoires de l'eau et la décentralisation. La gouvernance de bassin versant ou les limites d'une évidence. Développement durable et territoires, dossier 6). http:// developpementdurable.revues.org/1742 ; DOI : 10.4000/developpementdurable.1742

Ministerio de Agricultura y Desarrollo Rural MADR, 2014. Anuario estadístico del sector agropecuario 2013. Resultados, evaluaciones agropecuarias municipales. 304 p.

Ministerio de Ambiente, 1993. Ley 99 de diciembre 22 de 1993. Diario Oficial nº 41.146, Bogotá. Ministerio de Ambiente y Desarollo Social (MADS) et CORPOBOYACÁ, 2013. Reglamento interno de la Mesa de trabajo permanente de acompañamiento al proyecto piloto para la implementación de la política nacional de la gestión integrada del recurso hídrico en un humedal de alta montaña - lago de Tota. 6 p.

Ministerio de Ambiente y Desarollo Social (MADS), 2015. Decreto 1076 de 2015.

Niño A. M. M., Zambrano, B. A. C. 2011. Uso de la fauna silvestre del Lago de Tota. Peces, herpetos, aves y mamíferos. Ambiente y Desarrollo, vol. 13, n² 25, 19 p.

Offner J.-M., Pumain D., 1996. Réseaux et territoires. Éditions de l'Aube, 281 p.

Orozco Arcila L., Mejia Alfonso S., Reyes Zambrano P., 2012. Foro lago de Tota: claridad y soluciones sostenibles para su conservación aquitania, cuítiva y Tota. Semana nacional de la ciencia, la tecnología y la innovación. Boyacá, 67 p.

Pérez Preciado A., 1975. Tota, más que un lago es un conflicto. Colección Indispensable, Bogotá. Pontificia Universidad Javeriana, 2005. Plan de Ordenación y Manejo de la Cuenca del Lago de Tota. Convenio número 038 de 2004. CORPOBOYACA -PUJ

Raymond P., 1990. El lago de Tota ahogado en cebolla: estado socioeconomico de la cuenca cebollera del lago de Tota. Pontificia Universidad Javeriana, $176 \mathrm{p}$. 
Salles D., Leroy P., 2013. Gouvernance environnementale. In Barbier R., Blondiaux L., Chateauraynaud F., Fourniau J.-M., Lefebvre R., Neveu C., Salles D. eds., Dictionnaire critique et interdisciplinaire de la participation. Paris GIS Démocratie et Participation, http://www.dicopart.fr/ $\mathrm{fr} /$ dico/gouvernance-environnementale

Scarwell H. J., Kergomard, C., Laganier R., 2008. Environnement et gouvernance des territoires : enjeux, expériences, et perspectives en région Nord-Pas de Calais. Presses Universitaires du Septentrion, 388 p.

Theys J., 2002. La gouvernance, entre innovation et impuissance. Le cas de l'environnement. Développement durable et territoires, dossier 2. http://developpementdurable.revues.org/1523 ; DOI : 10.4000/developpementdurable.1523

Torre A., Aznar O., Bonin M., Caron A., Chia E., Galman M., Lefranc C., Melot R., Guerin M., Jeanneaux P., Kirat T., Paoli J.C., Salazar M.I., Thinon P., 2006. Conflits et tensions autour des usages de l'espace dans les territoires ruraux et périurbains. Le cas de six zones géographiques françaises. Revue d'Économie Régionale \& Urbaine, (3), p. 415-453. http://www.cairn.info/revue-deconomie-regionale-et-urbaine-2006-3-page-415.htm ; DOI : 10.3917/reru.063.0415

Torre A., 2011a. Les processus de gouvernance territoriale. L'apport des proximités. POUR, $\mathrm{n}^{\circ}$ 209-210, p. 114-122. http://www.cairn.info/revue-pour-2011-2-page-114.htm DOI : 10.3917/ pour.209.0114

Torre A., 2011 b. Du bon usage des conflits ! L'expression des désaccords au cœur des dynamiques territoriales. Métropolities. eu, 5 p. http://www.metropolitiques.eu/Du-bon-usage-des-conflitsL.html

Torre A., 2015. Gouvernance territoriale et conflits d'usages. WikiTerritorial du CNFPT, http:// www.wikiterritorial.cnfpt.fr/xwiki/wiki/econnaissances/view/NotionsCles/ Gouvernanceterritorialeetconflitsdusages

Tratnjek B., Laslaz L., 2011. Les espaces protégés : des territoires de conflits ? Café Géographique. http://www.Cafe-geo.net/article.php3?id_article $=2178$

Young, O., 2009. Governance for the Environment, New Perspectives. Presse universitaire de Cambridge, pp. 12-40.

Waves, 2016. Contabilidad ambiental y económica para el agua: caso piloto para la cuenca del lago de Tota. $84 \mathrm{p}$.

\section{NOTES}

1. Dans le cadre de ce travail, le bassin versant du lac de Tota est entendu comme un territoire à part entière car «bien qu'il s'agisse d'un objet géographique présenté comme naturel, le bassin versant n'en demeure pas moins une forme de découpage de la nature qui renvoie toujours à une vision particulière du territoire et de sa finalité » (Ghiotti, 2006).

2. C'est notamment le cas de Sogamoso, deuxième ville du département de Boyacá. Elle se situe à 20 kilomètres en aval du lac et compte 115000 habitants.

3. Selon la loi 99 de 1993, les zones de páramos, sub-páramos, les sources d'eau et les zones de recharge des aquifères seront l'objet d'une protection spéciale.

4. Avant la CAR, il existait également l'INDERENA (Instituto Nacional de Recursos Naturales Renovables y del Ambiente) mis en place dans les années 1960.

5. POMCA (Planes de Ordenación y Manejo de Cuencas Hidrográficas). Soulignons d'ailleurs que ce plan d'aménagement a été établi à travers une convention avec l'Université Javeriana ( $\left.{ }^{\circ} 083 / 2004\right)$, ce qui montre bien l'implication du monde académique dans la gouvernance du lac. 
6. «Tota, plus qu'un lac, un conflit ».

7. Mouvement civique pro lac de Tota et de son bassin versant.

8. Gestion environnementale intégrale du bassin hydrographique du Lac de Tota.

9. Le bureau permanent de travail du lac de Tota.

10. Le conseil du bassin versant du lac de Tota.

11. Suite au décret 1640 de 2012 (aujourd'hui décret 1076 de 2015).

12. Citation de Felipe Velasco.

\section{ABSTRACTS}

The purpose of this work is to show that conflicts play an essential role in the processes of territorial environmental governance. It is based on the case study of the watershed of Lake Tota, the largest lake in Colombia, which is now threatened by the development of several economic activities, and in particular by the intensive cultivation of spring onion. In a context of overexploitation and pollution of the lake's water, environmental conflicts have been exacerbating in recent years between actors the different interests assert themselves a little more each day: rural communities, environmental authorities, environmental associations (Montecito). Revealing the profound changes in this territory, these conflicts will help to take into account environmental problems and allow the various actors to influence decisions. However, the introduction of new forms of coordination between these actors remains an important source of opposition. This is evidenced by the competition between two governance structures (Mesa de trabajo Permanente and Consejo de Cuenca) which highlights the power relations between public authorities and civil society.

Cette contribution a pour objectif de montrer que les conflits jouent un rôle essentiel dans les processus de gouvernance territoriale de l'environnement. Elle s'appuie sur l'étude de cas du bassin versant du lac de Tota, plus grand lac de Colombie, aujourd'hui menacé par le développement de plusieurs activités économiques, et en particulier par la culture intensive de la ciboule. Dans un contexte de surexploitation et de pollution de l'eau du lac, on assiste depuis quelques années à l'exacerbation de conflits environnementaux entre des acteurs dont les intérêts divergents s'affirment un peu plus chaque jour: communautés rurales, autorités environnementales, association environnementale (Montecito). Révélateurs des mutations profondes de ce territoire, ces conflits vont contribuer à la prise en compte des problèmes environnementaux et permettre aux différents acteurs d'infléchir des décisions. Toutefois, la mise en place de nouvelles formes de coordination entre ces acteurs reste encore une importante source d'oppositions. En témoigne la concurrence entre deux structures de gouvernance (Mesa de trabajo Permanente et Consejo de Cuenca) qui met en lumière les rapports de pouvoir entre les pouvoirs publics et la société civile.

Este trabajo tiene por objetivo mostrar que los conflictos juegan un rol esencial en los procesos de gobernanza territorial del ambiente. Se apoya en el estudio de caso de la cuenca hidrográfica del lago de Tota, el más grande de Colombia, actualmente amenazado por el desarrollo de varias actividades económicas, particularmente por el cultivo intensivo de cebolla larga. En un contexto de sobrexplotación y de contaminación del lago, se observa, después de algunos años, la intensificación de conflictos ambientales entre los actores, donde los distintos intereses se 
afirman un poco más cada día: comunidades rurales, autoridades ambientales, asociación ambiental (Montecito). Se observa que estos conflictos no son eventos únicamente negativos, pues son también una plataforma para tomar la palabra. Revelador de los profundos cambios en este territorio, estos conflictos van a contribuir a la toma en cuenta de problemas ambientales y permitir a los diferentes actores influir en las decisiones. Sin embargo, la introducción de nuevas formas de coordinación entre estos actores sigue siendo una importante fuente de oposición. Esto se pone en relieve en la competencia entre dos estructuras de gobernanza (Mesa de trabajo Permanente y Consejo de Cuenca) que destaca las relaciones de poder entre los poderes públicos y la sociedad civil.

\section{INDEX}

Palabras claves: gobernanza territorial del ambiente, conflicto de uso, agricultura intensiva, cuenca hidrográfica del lago de Tota, Colombia

Keywords: territorial environmental governance, conflict of use, intensive agriculture, Lake Tota hydrographic basin, Colombia

Mots-clés: gouvernance territoriale de l'environnement, conflit d'usage, agriculture intensive, bassin versant du lac de Tota, Colombie

Subjects: Sur le Champ - Sur le Terrain

\section{AUTHORS}

\section{DAVID LEROY}

David Leroy, david4leroy@hotmail.fr , est titulaire d'un doctorat en Environnement et Sociétés obtenu à l'Université Toulouse Jean Jaurès, GEODE UMR 5602 CNRS. Il a publié récemment :

- Blot F., Peltier A., Antoine J.-M., Angeliaume-Descamps A., Leroy D., Maire E., Molina L.E., Rivero J.C., 2015. Vulnérabilités liées à l'eau dans les Andes vénézuéliennes : influences des relations sociétés/hydrosystèmes dans le cas de Santa-Cruz-de-Mora. L'Ordinaire des Amériques, $\mathrm{n}^{\circ} 218$. https://orda.revues.org/1905

- Angéliaume-Descamps A., Blot F., Leroy D., 2013. Dynamique récente des relations aux zones humides des páramos andins vénézuéliens : entre fonctionnalisme et mystique. Géocarrefour, 88(4), p. 285-298. https://geocarrefour.revues.org/9278

- Leroy D., Angéliaume-Descamps A., Antoine J.-M., Blot F., Maire M., Peltier A., 2013.

Représentation et pratiques autour de la ressource en eau des producteurs maraîchers des Andes vénézuéliennes. Vertigo, varia, vol. 13, n 1, avril 2013. http://vertigo.revues.org/13356

\section{ALVARO MARTÍN GUTIÉRREZ MALAXECHEBARÍA}

Alvaro-Martín Gutiérrez-Malaxechebaría, amgutierrezm@udistrital.edu.co ,PhD Estudios Ambientales y Rurales, Mg en Ingeniería Ambiental, Ingeniero Civil, Profesor tiempo completo ingeniería ambiental, Facultad de Estudios Ambientales y Rurales, Universidad Distrital Francisco José de Caldas. Il a publié récemment:

- Forero-Álvarez J., Rodríguez-Bernal C. S., Gutiérrez-Malaxechebarría A., Nieto-Méndez A., 2016. Eficiencia económica y potencialidades para superar la pobreza de la agricultura familiar colombiana. En El desarrollo equitativo, competitivo y sostenible del sector agropecuario en Colombia. Bogotá, Banco de la República de Colombia, CAF.

- Gutiérrez-Malaxechebarría A., Rodriguez Zambrano Y., Ospina Hoyos L., 2015. Propuesta metodológica de priorización de areas para conservación de cuencas. Validación en río Caquinal, 
Fómeque, Cundinamarca, Colombia. Revista de Investigación Agraria Y Ambiental, 6(1), p. 199-214. - Gutiérrez-Malaxechebarría A., 2014. Formal and informal irrigation in the Andean countries. An overview. Cuadernos de Desarrollo Rural (International Journal of Rural Development), 11(74), p. 75-99. doi:10.11144/javeriana.CRD11-74.fiac

\section{JEAN-MARC ANTOINE}

Jean-Marc Antoine, antoine@univ-tlse2.fr, est Maître de conférences HDR, Université de Toulouse Jean Jaurès, GEODE UMR 5602, Directeur du Département de Géographie, Aménagement, Environnement. Il a publié récemment :

- Blot F., Peltier A., Antoine J.-M., Angeliaume-Descamps A., Leroy D., Maire E., Molina L.E., Rivero J.C., 2015. Vulnérabilités liées à l'eau dans les Andes vénézuéliennes : influences des relations sociétés/hydrosystèmes dans le cas de Santa-Cruz-de-Mora. L'Ordinaire des Amériques, nº 218. https://orda.revues.org/1905

- Daupras F., Antoine J.-M., Becerra S., Peltier A., 2015. Analysis of the robustness of the French flood warning system: a study based on the 2009 flood of the Garonne River. Natural Hazards, vol. 75, $\mathrm{n}^{\circ} 1$, p. 215-241.

- Becerra S., Peltier A., Antoine J.-M., Labat D., Chorda J., Ribolzi O., Daupras F., Dartus D., 2013. Comprendre les comportements face à un risque modéré d'inondation. Étude de cas dans le périurbain toulousain (Sud-Ouest de la France). Hydrological Sciences Journal, 58, 5, p. 945-965.

\section{ALEXANDRA ANGÉLIAUME-DESCAMPS}

Alexandra Angéliaume-Descamps, angéliau@univ-tlse2.fr , est Enseignant chercheur, GEODE UMR 5602, Université de Toulouse Jean Jaurès. Elle a publié récemment :

- Blot F., Peltier A., Antoine J.-M., Angeliaume-Descamps A., Leroy D., Maire E., Molina L.E., Rivero J.C., 2015. Vulnérabilités liées à l'eau dans les Andes vénézuéliennes : influences des relations sociétés/hydrosystèmes dans le cas de Santa-Cruz-de-Mora. L'Ordinaire des Amériques, $\mathrm{n}^{\circ} 218$. https://orda.revues.org/1905 - Angéliaume-Descamps A., Gutiérrez Malaxechebarría A. M., 2013. Modèles d'irrigation dans les petits systèmes maraîchers des Andes vénézuéliennes et colombiennes. In Angéliaume-Descamps A., Corrales E., Jamirez Juarez R., Tulet J.-C. (coord.), La petite agriculture familiale des Hautes Terres tropicales. Colombie, Mexique, Venezuela. L'harmattan, p. 193-227, 336 p.

- Leroy D., Angéliaume-Descamps A., Antoine J. M., Blot F., Maire M., Peltier A., 2013.

Représentation et pratiques autour de la ressource en eau des producteurs maraîchers des Andes vénézuéliennes. Vertigo, varia, vol. 13, n 1, avril 2013. http://vertigo.revues.org/13356 